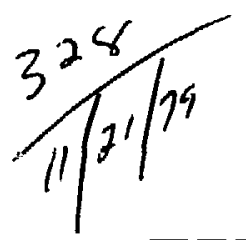

\title{
MASTER
}

DR. 322

\section{TOWARDS UNIFIED PERFORMANCE MEASURES FOR EVALUATING NUCLEAR SAFEGUARD SYSTEMS: MATHEMATICAL FOUNDATIONS AND FORMAL COMPARISON OF EXISTING MODELS}

G. C. Corynen

May 15, 1979

Work performed under the auspices of the U.S. Department of Energy by the UCLLL under contract number W-7405-ENG-48.

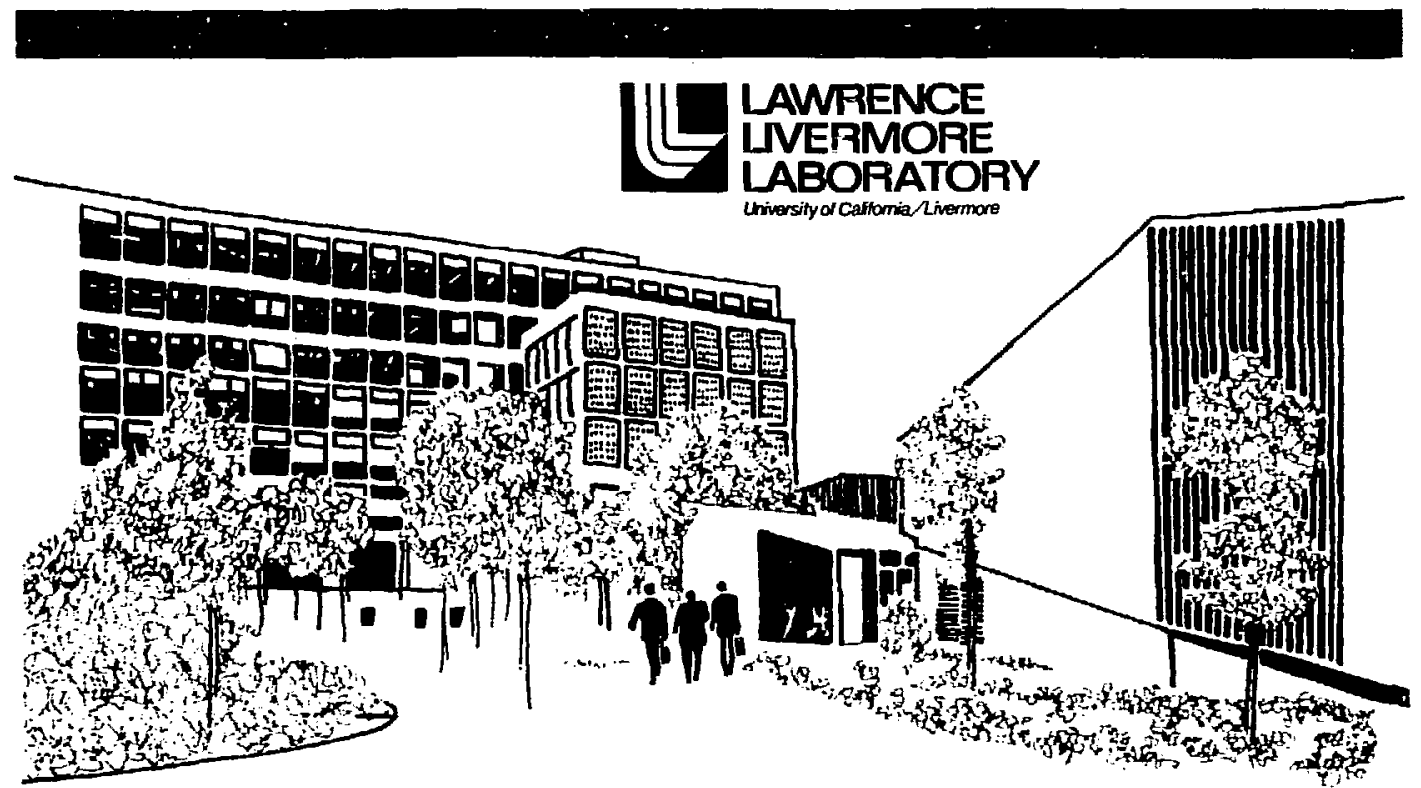




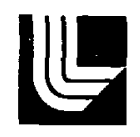

LAWRENCE LIVERMORE I_ABORATORY

University of Cahtornia Livermore, Calitornia 94550

UCRL-52793

\title{
TOWARDS UNIFIED PERFORMANCE MEASURES FOR EVALUATING NUCLEAR SAFEGUARD SYSTEMS: MATHEMATICAL FOUNDATIONS AND FORMAL COMPARISON OF EXISTING MODELS
}

\author{
G. C. Corynen
}

Manuscript Date: May 15, 1979

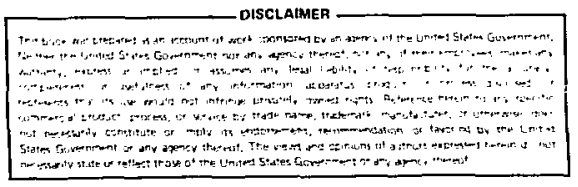




\section{CONTENTS}

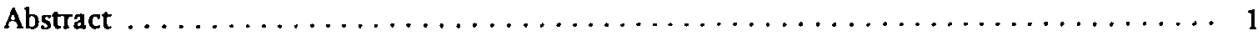

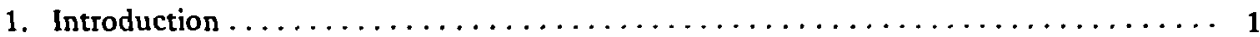

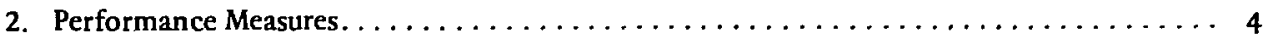

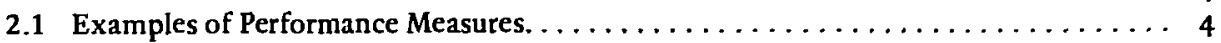

2.2 Performance Measures for Indexed or Parametrized Systems $\ldots \ldots \ldots \ldots \ldots \ldots$

2.3 Performance Measures for Systems Consisting of

Interconnected Subsystems. . . . . . . . . . . . .

3. Deterministic Analysis of Safeguard Systems $\ldots \ldots \ldots \ldots \ldots \ldots \ldots \ldots \ldots \ldots \ldots \ldots$

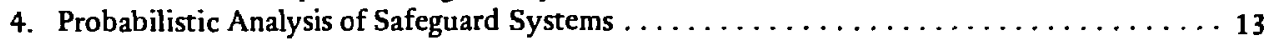

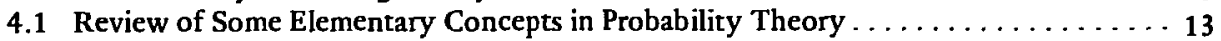

4.2 The Detection Subsystem as a Parametrized Collection of

Random Variables. . . . . . . . . . . . . . . . . . . 15

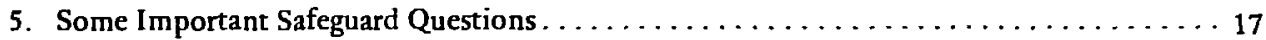

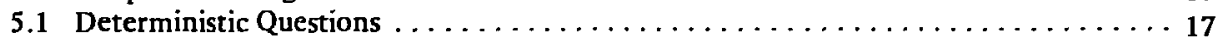

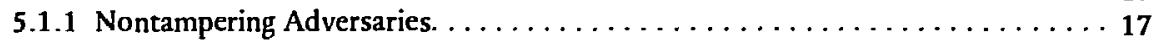

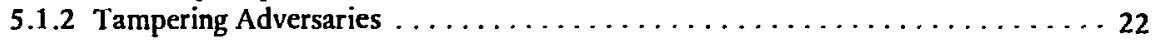

5.2 Probabilistic Questions . . . . . . . . . . . . . . . . . . . 24

6. The Structured Assessment Approach (SAA) Analyzed in

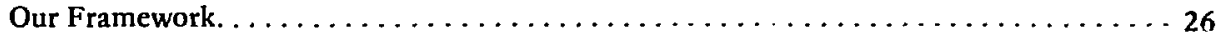

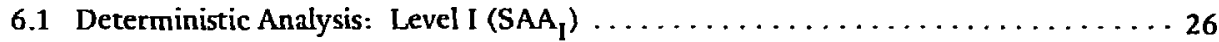

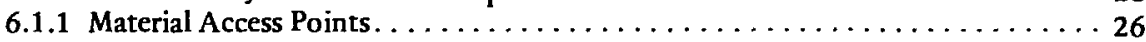

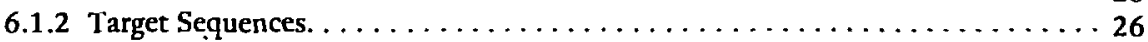

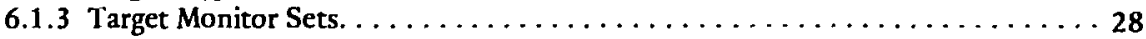

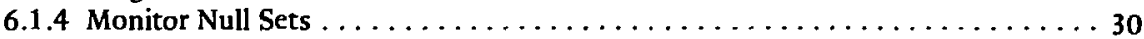

6.1.5 Summary of SAA in Our Framework, and Some

Qualitative Performance Measures.................... 31

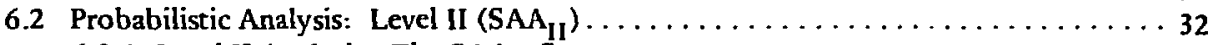

6.2.1 Level II Analysis: The SAA $_{\text {II }}$ System. $\ldots \ldots \ldots \ldots \ldots \ldots \ldots \ldots \ldots \ldots \ldots \ldots \ldots$

6.2.2 Adversaries Who Exploit Their Knowledge of the

Plant Operating Modc

7. The Systems Vulnerability Assessment Method (SVAM) Analyzed

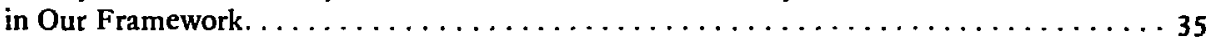

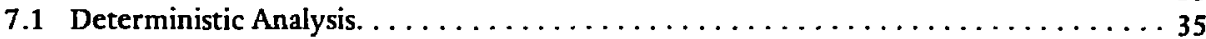

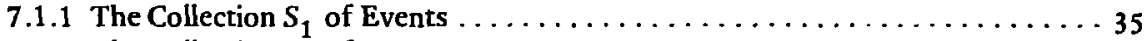

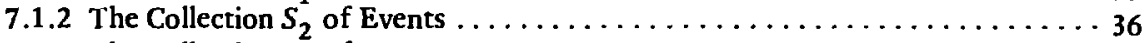

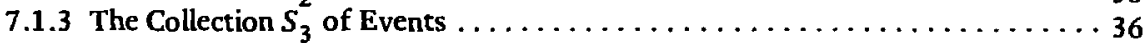

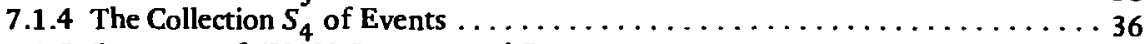

7.1.5 Summary of SVAM Outputs, and Some

Deterministic Performance Measures. . . . . . . . . . . . . . 36

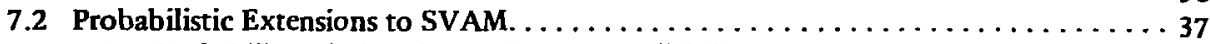

7.2.1 Probability of Diversion and System Reliability as Safeguard Performance Measures. . . . . . . . . . . . . 38

7.3 Probabilistic Performance Measures Associated with

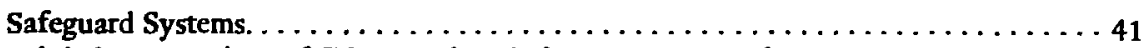

8. Deterministic Comparison of SVAM and SAA in Our Framework $\ldots \ldots \ldots \ldots \ldots \ldots$

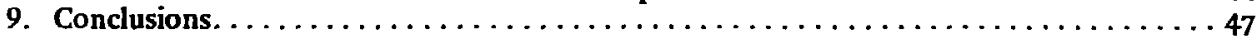

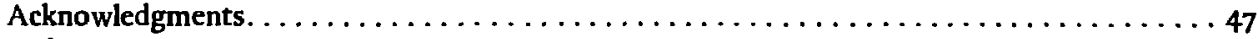

References. . . . . . . . . . . . . .

Appendix. The Safeguard System as a Structured Object. . . . . . . . . . . . . 49 


\title{
TOWARDS UNIFIED PERFORMANCE MEASURES FOR EVALUATING NUCLEAR SAFEGUARD SYSTEMS: MATHEMATICAL FOUNDATIONS AND FORMAL COMPARISON OF EXISTING MODELS
}

\begin{abstract}
An important step in the analysis of large-scale systems is the development of economical and meaningful measures of system performance. In the literature on analysis of nuclear safeguard systems, many performance measures have been advanced, but their interrelationships and their relevance to overall system objectives have not received attention commensurate with the effort spent in developing detailed safeguard models. The work reported here is an attempt to improve the development and evaluation of performance measures for nuclear safeguard systems. This work falls into three main areas. First, we develop a new framework which provides an initial basis for defining and structuring performance measures. To demonstrate that this framework allows a clear understanding of the purposes of nuclear safeguard systems, we employ it to state various safeguard questions clearly and concisely. The framework reflects the rough subsystem structure of safeguard systems-the detection and response subsystems-and formally accommodates various safeguard models. We especially emphasize two of these models which are under development at Lawrence Livermore Laboratory, the structured assessment approach (SAA) and the systems vulnerability assessment method (SVAM). Second, we examine some performance measures presently used in the nuclear safeguards area and in reliability theory in general. Some of these we accept and modify to obtain system performance measures that are an additive combination of subsystem performance measures, a very convenient form indeed. Others we reject as impractical and meaningless. Finally, we determine some common features between the SAA and SVAM models by formally comparing these models in our framework.
\end{abstract}

\section{INTRODUCTION}

The safeguarding of nuclear and other precious materials has been a difficult problem for a long time. Recently, the nuclear safeguarding problem has been the subject of various "systematic" investigations, ranging from extremely detailed analyses of nuclear diversion or theft scenarios to highly aggregated analyses where the social implications of malevolent nuclear acts are examined. A principal objective for these analyses is to develop standards that could be used by the Nuclear Regulatory Commission (NRC) in licensing nuclear facilities. The definition of such standards, and verification of compliance with them, requires an economical and practical set of performance criteria or measures by which the "hardness" of nuclear facilities to diversion, sabotage, or theft attempts can be judged. The development of such performance measures obviously requires a good understanding of nuclear facilities and their safeguard systems. ${ }^{1}$

At Lawrence Livermore Laboratory we have recently become interested in determining whether the state of the art in safeguards research is sufficiently mature to develop such performance measureš and reach some final conclusions from ongoing safeguard analyses. A natural first step in 
such a determination would be to survey the nuclear safeguards field and simply report a list of performance measures presently used or contemplated. We started such an effort but soon realized that it would not serve the optimistic goals for which it was intended, for several reasons. First, the term "performance measure" has different meanings, not only in the nuclear safeguards area, but in the systems analysis business generally. Some analysts interpret "performance measure" as a performance level to be met by a "good" design. Others use it to indicate a specific action to be taken in order to improve a system's performance. Yet others use performance measures as measuring sticks by which the quality of a given design can be evaluated.

A second reason why our goal was difficult to reach with a surveying approach was that some of these measures were developed in an ad hoc manner. In a recent collection of reports, ${ }^{2}$ for instance, several objectives for nuclear safeguards were proposed, and numerous performance measures were recommended and discussed. Although this was a good initial attack on the problem, the relationship or structure between these measures, and between the measures and their associated objectives, was not clear. As a result, it was difficult to rank the importance of these measures, and to recommend which would be good candidates for use in NRC regulations.

A third reason why further work was needed before definitive conclusions could be reached with the various performance measures proposed was that it was not clear how these measures could be computed, using various safeguard models under development, for safeguard systems of potential interest to the NRC.

Finally, and what is probably the most serious objection, some of these measures could not withstand close scrutiny when their exact meaning, purpose, and defensibility were questioned.

Our first step was consequently redirected towards the development of a simple and concise model of a general safeguard system-a model that could be used to state a large collection of safeguard questions clearly, that would provide an underlying structure for development of performance measures, and that would allow the discussion and comparison of other models already developed and the results obtained with them. The purpose of this paper is to describe the model we have developed and give some of the results obtained with it. We shall show that these results considerably clarify our understanding of what performance measures are and direct us in determining which measures may be useful in aggregating numerous and detailed modeling results into a few performance statements.

Most recent work in safeguard systems analysis has been of a mathematical, statistical, and computational nature, and has dealt with "sets of things"-adversary action sets, consequence sets, target sets, and the like. Thereforc, we have chosen mathematics as a foundation for our entire discussion, with particular emphasis on set theory.

Even though a lot of safeguard systems analysis work has been accomplished in recent years, it is astonishing that the language used in recent reports, and even in glossaries of terms and concepts, is still quite vague, and sometimes inconsistent. Although we are not interested in singling out works and authors for criticism, we should note that some of these works also report several conclusions reached with the use of complex computer algorithms. Computing machines, however, do not tolerate ambiguity, inconsistency, or fuzziness in their programs. Hence, to reconcile these incompatible points of view-the computer's insistence on precise terminology and the analyst's accustomed neglect of it-a lot of definitional work must have taken place implicitly during the programming stages, and this work was probably either too complex or too artistic to report. A major purpose of this paper is to develop a framework with which some of these implicit processes can be made more explicit. Besides formalizing some notions which may have been informally commonplace, going through another descriptive experience of various safeguard questions with mathematics as a vehicle would be an instructive exercise. Even for those analysts who are quite familiar with the safeguards problem, new questions may surface, and perhaps some inconsistencies in past analyses may be revealed. We should note here that we do not lay claim to presenting an infinitely accurate account of the "safeguards problem," only that we discuss some aspects of it precisely and consistently. 
While mathematics is unforgiving of vagueness and fuzziness, it is only a Leibnitzian construct consisting exclusively of synthetic truths, and it cannot tell us anything about a "physical reality" without the assistance of epistemic relations which append to it the appropriate semantics about the real world being modeled. Hence, what we say in certain places of this report may in fact be complete nonsense, but it will be precise nonsense, which will be prepared for immediate surgery. While some concepts developed by other authors will become clearer when seen in a more formal format, others will become more confused because the original authors may doubt the meaning which surfaces once they see their concepts expressed mathematically. All this is very healthy. We thus advise the nontechnical readers not to be distracted by the mathematics, but to look through it, and concentrate on the more informal discussions which surround it. As we used it in this work, mathematics was not an end in itself, but a means to crystallize ideas and activities.

We have accomplished three major things in this repart. First, we have developed a new framework which provides an initial basis for defining and structuring performance measures. We demonstrate that it allows a clear understanding of the purposes of nuclear safeguard systems by employing it to state various safeguard questions clearly and concisely. The framework also reflects the rough subsystem structure of safeguard systems-the detection and response subsystems-and formally accommodates various safeguard models. Two of these models are presently under development at Lawrence Livermore Laboratory under the names of the structured assessment approach (SAA) and the systems vulnerability assessment method (SVAM), and will be especially emphasized. Second, we have examined some performance measures presently used, not only in the nuclear safeguards area, but in reliability theory in general. Some of these we have modified to obtain system performance measures that are an additive combination of subsystem measures, a very convenient form indeed. Others we have rejected as impractical and meaningless. Finally, we have determined some common features between the SAA and SVAM models by formally comparing these models in our framework.

The paper we have produced may appear overwhelming, at least in its quantity of topics, explanations, introductions, developments, etc. This is because. during prior review of it, some reviewers expressed the opinion that it should be relatively self-contained, explanatory, tutorial in nature, and not merely addressed to systems theorists or mathematicians. Therefore, it may appear verbose to some, brief and abstract to others, and altogether unintelligible to others. We beg the reader's understanding of these basic constraints imposed on an author genuinely interested in bridging the gap between theoretical systems science and the practical problems faced by safeguard engineers.

The report consists of eight chapters and is organized as follows. In Chapter 2, we define what is meant by "performance measures" and identify three basic types: qualitative, quantitative, and classificatory measures. We then show how performance measures can be identified with general parametrized systems, and with systems consisting of well-defined subsystems whose individual performance is also of interest. Then we develop a formal deterministic model of safeguard systems in Chapter 3, and a probabilistic model in Chapter 4. In Chapter 5 we express some important safeguard questions in this framework. Next, in Chapter 6, we analyze the SAA model in our framework, and in Chapter 7 we analyze the SVAM model in a similar manner. Then we compare SAA and SVAM in Chapter $B$ and formally demonstrate that SVAM is equivalent to parts of SAA. Finally, we provide some conclusions in Chapter 9 . 


\section{PERFORMANCE MEASURES}

The principal theme of this paper is "performance measures." In this chapter, we intend to precisely convey the mathematical meaning of this theme.

Given a set $S$ of systems or objects $s$, the purpose of a performance measure ${ }^{3}$ is to extract some essential characteristics from elements $s \in S$. These characteristics are members of another set $V$, the performance set, which includes all possible values of the features or characteristics of interest: the performance values for objects in S. We have previously described (in Ref. 1, Appendix A) three types of properties which play an important role in science and engineering: quantitative, qualitative, and classificatory properties. Performance measures may be taxonomized in an analogous manner, and in this general setting are simply relations $\Phi$ between $S$ and $V$. More specifically, $\Phi \subset S \times V$. With each point $s \in S$, the domain of the performance measure, is thus associated a set of performances $\Phi(s) \subset V$ (Fig. 2-1), and $\Phi$ is then referred to as a qualitative performance measure. If $\operatorname{PAR}(V)$ is a partition of $V$ and if every set $\Phi(s), s \in S$, is contained in one and only one element of $\operatorname{PAR}(V)$, then $\Phi$ is called a classificatory performance measure. In this case, the performances or features of every element $s \in S$ are classified in one and only one class of $\operatorname{PAR}(V)$. If $V$ is a set of real numbers and $\Phi$ is a function, then $\Phi$ is a quantitative performance measure. In that case, $\Phi$ associates with each $s \in S$ a real number $\Phi(s) \in V$, the performance of $s$. By definition, any performance measure is qualitative if and only if (iff) it is not quantitative. Among these three basic types of performance measures, quantitative measures are believed to be most important in safeguard systems analysis.

\subsection{EXAMPLES OF PERFORMANCE MEASURES}

In this section we present some general examples of performance measures. Numerous examples of safeguard-system performance measures will be discussed in later chapters throughout this report.

Consider the simple example where $S$ is a set of systems each of which generates an output random variable $Y_{s}, s \in S$ (Fig. 2-2). Then one is often interested not only in the statistical properties of $Y_{s}$ but also in how to represent its distribution function $F_{Y_{s}}$ "economically." If the shape of these distributions is well behaved as in Fig. 2-3 (e.g., symmetric, small variance, etc.), the assumption is often made that the expected value $E\left(Y_{s}\right)$ satisfactorily summarizes the statistical characteristics of $Y_{s}, s \in S$ (in fact, this assumption is often made even when almost nothing is known about the distributions). In this case, the performance of each $s$ is the expected value $E\left(Y_{s}\right)$. Hence the evaluation map $\Phi: S \rightarrow V$ is a quantitative performance measure which is defined by the following condition: For all $s \in S, \Phi(s)=E\left(Y_{s}\right) \in V=R$ (the real numbers).

Next, consider an example where "performance" is taken more literally. Let $S$ consist of all $n$-dimensional linear control systems $X=A_{s} X+B_{s} u$, where the control $u$ is an $n$-vector and $A_{s}$ and $B_{s}$ are $n \times n$ matrices associated with system $s \in S$. The analyst is often interested not only in the trajectory $X_{s}$ (state solution of $s$ ) when the system $s$ is driven with a predetermined input $u$, but also in determining whether the system gets to some final target $x_{f}$ from some fixed initial state $x_{0}$ by some time $t_{f}$, and how much it "costs" to get there. Consider some real-valued $\operatorname{cost} J\left(X_{s}, x_{f}, t_{f}\right)$. Then it makes sense to judge the performance of any $s \in S$ by the number $J\left(X_{s}, x_{f}, t_{f}\right)$. Therefore, $\Phi: S \rightarrow V=R$ is again a quantitative performance measure defined by:

For all $s \in S, \Phi(s)=J\left(X_{s}, x_{f}, t_{f}\right)$.

Next, consider the physical case where the input-output $(I / O)$ behaviors of some physical systems are of interest. These could be electronic circuits, for instance (Fig. 2-4), whose currentvoltage relationships $(I / V)$ are being observed with an ammeter $M$ with range $X_{M}$ and a voltmeter with 
range $Y_{M}$ yielding, for every system under study, a finite set of performance pairs $\left\{(x, y)_{n} \in X_{M} \times Y_{M}\right.$, $n=1,2, \ldots, N\}$. If the collection of systems (circuits) is represented by the set $S$ and each system is labeled as some $s \in S$, then the performance measurement process can be represented mathematically

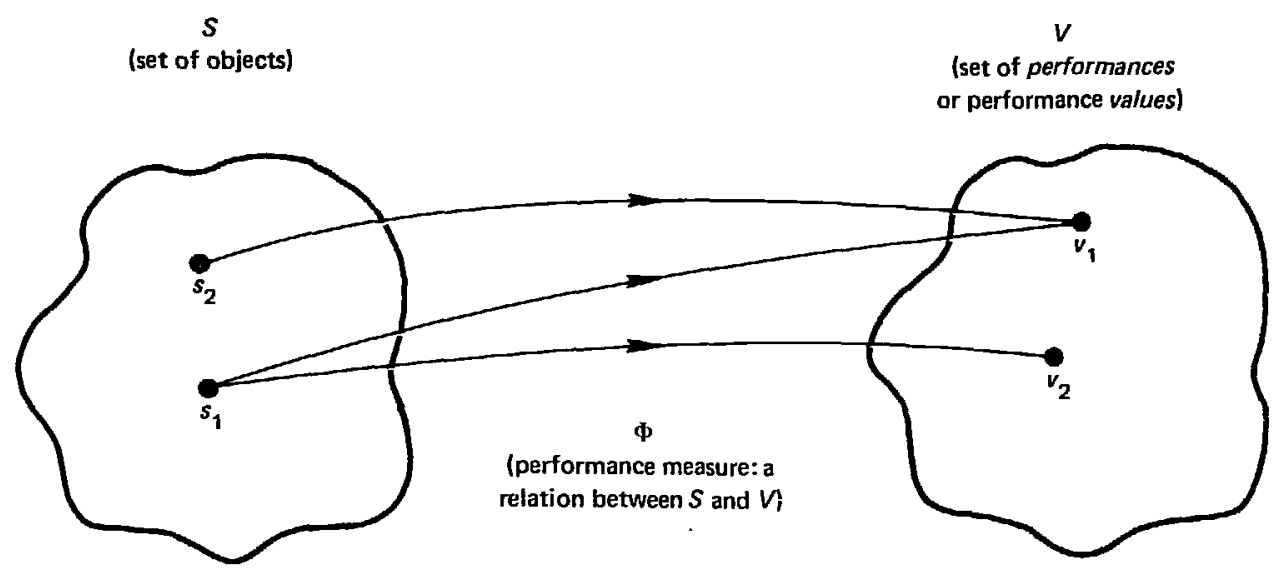

Fig. 2-1. The concept of a performance measure as a relation $\Phi$ between a set of objects $S$ and a set of performances $V$.
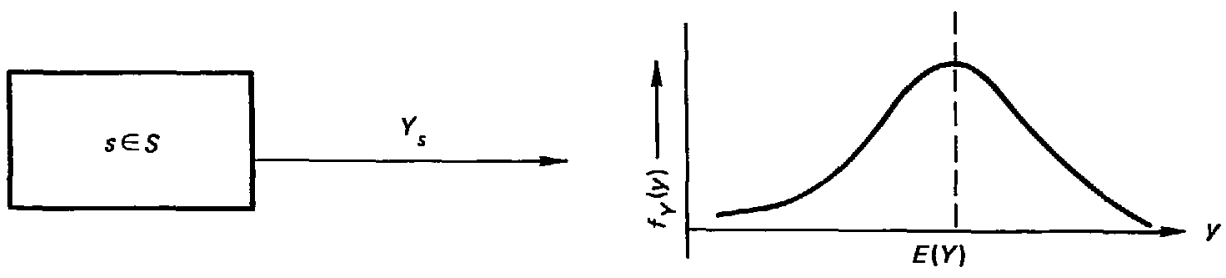

Fig. 2-2. A particular system $s \in S$ with output $Y_{5}$.

Fig. 2-3. The case where $Y$ is characterized by $E(Y)=\int_{R} y f_{Y}(y) d y$.

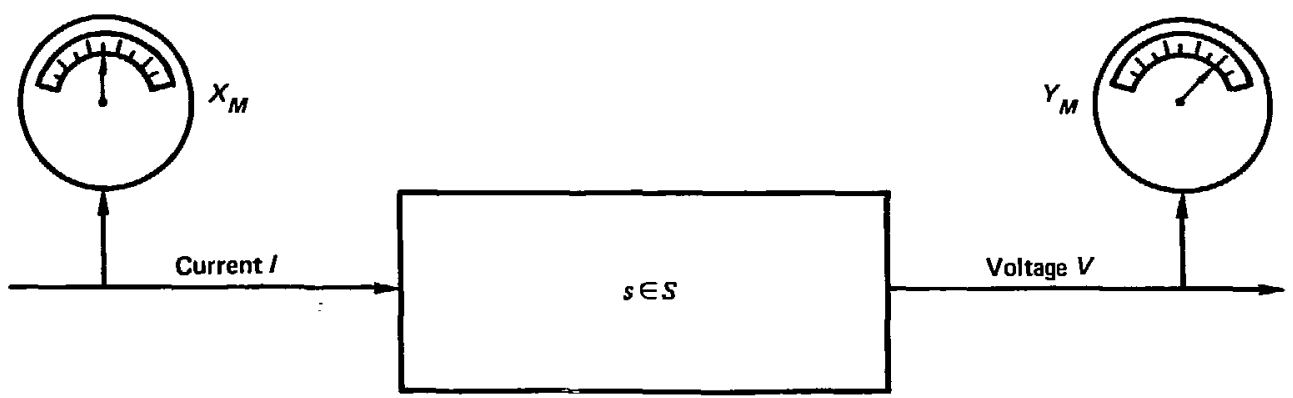

Fig. 2-4. A possible physical system of interest. 
as before by a map $\Phi: S \rightarrow V=2^{X_{M} \times Y_{M}}$ (the set of all relations on $X_{M} \times Y_{M}$ ), and for all $s \in S$, $\Phi(s)=\left\{\left(x_{5} y_{s}\right)_{n} \in X_{M} \times Y_{M}: n=1,2, \ldots, N(s)\right\}$. In this case, $\Phi$ is thus a qualitative performance measure.

Finally, consider the more specific problem where the fuel economy of some collection of vehicles is to be judged as low, medium, or high. The $S$ is the set of vehicles of interest, $V$ is a set of positive real numbers (the milcs-per-gallon or mpg values), and $\Phi: S \rightarrow V$ associates with each vehicle $s \in S$ its fuel economy $\Phi(s) \in V$ (in $\mathrm{mpg}$ ). If vehicles are to be classified as super economy (SE), economy (E), or gas guzzlers (GG), then $V$ can be partitioned into three sets $\operatorname{PAR}(V)=\left[V_{\mathbf{S E}}\right.$, $\left.V_{\mathrm{E}}, V_{\mathrm{GG}}\right\}$. Since the performance of each vehicle falls in only one element of $\operatorname{PAR}(V), \Phi$ is a classificatory performance measure.

An important remark should be made at this point. If the set of objects $S$ contains an incompletely specified object, it must contain all conceivable versions of that object. If we say that $S$ contains some system like that in Fig. 2-4 which is unknown except for the input and output spaces $X$ and $Y$, and the performance function is the $I / O$ relation $r_{s} \subset X \times Y$, i.e., for all $s \in S, \Phi(s)=r_{s} \in V$. then $S$ must contain all systems $s$ such that $\Phi(s) \in 2_{2} X X^{s}$ (the set of all relations $X \times Y$ ), because otherwise evaluation may be impossible. For instance, further measurement may reveal the exact structure (behavior) $r_{s}$, of some $s^{\prime}$ which was not included in $S$. Although $r_{s}, \in V, \Phi$ is not defined for $s^{\prime}$. In other words, $S$ must include all relevant possibilities.

The modeler thus expresses the important features of the objects in $S$ with a performance relation $\Phi$, and the !' represents the possible values of these features.

\subsection{PERFORMANCE MEASURES FOR INDEXED OR PARAMETRIZED SYSTEMS}

The development of performance measures for a system requires a good understanding of the structure of that system. In another major report, ${ }^{1}$ we have developed a foundation for formally structuring complex systems. The principal ingredient of that foundation is the concept of a "parametrized system."

Parametrized systems play an inportant role when the sensitivity of systems to changes in their internal, environmental, or input conditions is of interest. Parametrized systenic are also essential when very little is known about some of these conditions. If a system is sensitive to süch unknown conditions, several models may have to be developed, one for each posited condition. In nuclear safeguard systems, for instance, the sensitivity of undesirable social consequences to the type of adversary who is diverting nuclear materials may be very high. Because the likelihood that any given adversary may confront a given facility during some time period is very speculative, the consequences of nuclear material diversion by any of the various plausible adversaries cannot conveniently and accurately be aggregated into a holistic consequence statement. It is then useful, if not mandatory, to analyze social consequences separately for each different adversary type, and this type is then treated as a parameter.

When systems are parametrized, their performance measures are usually parametrized as well, as we shall see. It is thus important, at this point, to review what we mean by a parametrized system, and how performance measures for such systems are developed.

Definition. A parametrized system, represented by the set $s_{\Theta}=\left\{s_{\theta}: \theta \in \Theta\right\}$ is a function $\psi: \Theta \rightarrow S$ from a given set $\Theta$ (the parameter or index set) into another set $S$ (the set of systems). Since $s_{\Theta}$ is a map, the expression $s_{\Theta}(\theta)=s_{\theta}$ makes sense, and we call $s_{\theta}$ the system at parameter setting $\theta$. Many examples of such systems will be considered later in this report.

Consider the simple safeguard $5 y \operatorname{sis}$ sh shown in Fig. 2-5, where $U$ is a set of adversary strategies or action sequences, $\Omega$ is a set of system operating conditions, $Y$ is the set of physical outcomes of strategies $u$ (at the facility), and $W$ is the set of social consequences ("disutilities," for instance) of these outcomes. 
The outcomes $y \in Y$ and social consequences $w \in W$ of an adversary's attempting a given strategy $u \in U$ will depend on the operating condition, state, or constraints imposed on the system, and we treat these factors collectively as a parameter $\omega \in \Omega$. If we fix $\omega \in \Omega$, we might thus model the system as a function $\mathrm{SG}_{\omega}: U \rightarrow Y$ from $U$ to $Y$. Each parameter value $\omega \in \Omega$ thus gives rise to such a function. If we are interested in the sensitivity of the safeguard system to changes in $\omega$, we would incorporate this into our model by expressing it as an indexed or parametrized system $\mathbf{S G}_{\Omega}$ $=\left\{\mathrm{SG}_{\omega}: \omega \in \Omega\right]$.

In general, the performance measure $\Phi_{\Theta}$ for a parametrized system $s_{\Theta}$ is derived from performance measures associated with its elements $s_{\theta}$. The most obvious way in which this occurs is when a performance profile is considered. Starting with a measure $\Phi$ for the elements $s_{\theta} \in s_{\Theta}$, the performance profile of $s_{\Theta}$ is simply the collection of parameter-performance pairs $\Phi_{\Theta}\left(s_{\Theta}\right)=\{(\theta$, $\left.\left.\Phi\left(s_{\theta}\right)\right): \theta \in \Theta\right\}$, one pair for each parameter value $\theta$. In vulnerability analyses, for instance, the vulnerability characteristic of a system to a set of conditions or tbreats $\Theta$ is such a profile. For each threat $\theta \in \Theta$, the performance $\Phi\left(s_{\theta}\right)$ is listed in mathematical or graphical form.

In safeguard analyses, one might wish to allocate safeguard resources to various nuclear facilities according to some optimal allocation level determined for each facility. If we take Fig. 2-5, where SOC is a social utility function of physical consequences $y$ and attempts $u$, and $\omega$ is the parameter specifying the resource allocation level, we might consider the expected utility over attemptconsequence pairs as a criterion for setting this allocation level. One performance profile is then simply

$$
\Phi_{\Omega}\left(\mathrm{SG}_{\Omega}\right)=\left\{\left(\omega, E\left[\operatorname{SOC}\left(u, \mathbf{S G}_{\omega}(u)\right)\right]\right): \omega \in \Omega\right\}=\left\{\left(\omega, \Phi\left(\mathbf{S G}_{\omega}\right)\right): \omega \in \Omega\right\}
$$

where

$$
\Phi\left(\mathrm{SG}_{\omega}\right)=E[\operatorname{SOC}(u, \operatorname{SG}(u, \omega))]=\int_{U} \operatorname{SOC}(u, \operatorname{SG}(u, \omega)) p(u) d u,
$$

the expected value of a utility function SOC for parameter value $\omega$ with respect to the density function $p(u)$.

The optimal allocation point $\omega^{*} \in \Omega$ would then be a value of $\omega$ at which a maximum of that profile is reached. We then have an overall performance function $\psi$ (assume $\Omega$ is finite):

$$
\psi\left(\Phi_{\Omega}\left(\mathrm{SG}_{\Omega}\right)\right)=\underset{\omega \in \Omega}{\operatorname{MAX}}\left\{\Phi\left(\mathrm{SG}_{\omega}\right): \omega \in \Omega\right\} .
$$

Any value of $\omega$ associated wirh that maximum is an optimal value.

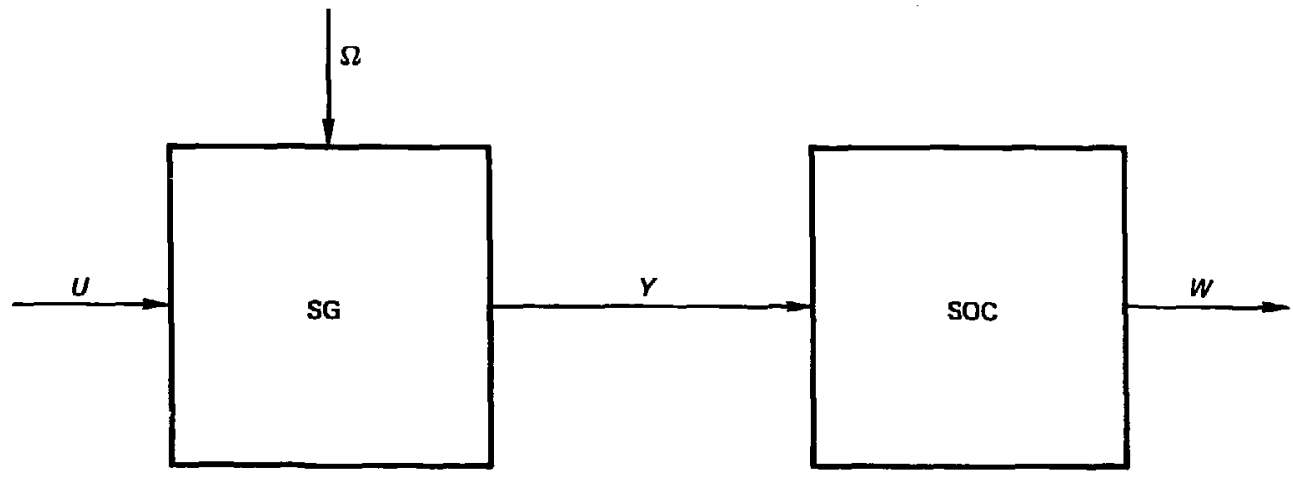

Fig. 2-5. A simple model of a safeguard system as a parametrized object. 


\subsection{PERFORMANCE MEASURES FOR SYSTEMS CONSISTING OF INTERCONNECTED SUBSYSTEMS}

We view a system as a collection of interact: subsystems, and have formalized this view in Ref. 1. When the performance of systems is considered, the most important system-analytic problem is the determination of system performance from the performances of its subsystems and their interactions with one another. More specifically, when we have a system $s \in S$ with a collection of subsystems $\left[s_{i} \in S_{i}: i \in I\right]$, several important questions arise. We may start with a system performance measure $\Phi: S \rightarrow V$ and address the performance assessment problem of first determining a reasonable set of subsystem performance measures $\Phi_{i}: S_{i} \rightarrow V_{i}$, and then describing the system performance $\Phi(s)$ from the assessed subsystem performances $\Phi_{i}\left(s_{i}\right)$ via a system structure function $\psi\left(\Phi_{1}, \Phi_{2}, \ldots, \Phi_{|I|}\right) *$ Clearly, $\Phi(s)=\psi\left(\Phi_{1}\left(s_{1}\right), \Phi_{2}\left(s_{2}\right), \ldots, \Phi_{|I|}\left(s_{|I|}\right)\right)$.

Or we may ask if subsystem measures $\left\{\Phi_{i}: i \in I\right\}$ can be developed which allow a simple form of the structure function $\psi$. For instance, the additive form $\psi\left(\Phi_{1}, \ldots, \Phi_{|I|}\right)=\sum_{i=1}^{|I|} a_{i} \Phi_{i}$ is often sought. In Chapter 7, we show in fact that if a safeguard system is viewed as consisting of two major subsystems $D$ (detection) and $R$ (response), then a probabilistic measure $\Phi$ for the system can be developed which is the sum of two measures $\Phi_{D}$ and $\Phi_{R}$ for $D$ and $R$, respectively. These measures themselves are shown to have an additive form of their own, a very convenient and useful formulation.

FI is the cardinality of $I$. 


\section{DETERMINISTIC ANALYSIS OF SAFEGUARD SYSTEMS}

In this chapter, we develop a formal deterministic model of a general safeguard system at a particular level of detail. We then use this model to generate some questions of relevance to the assessment of the performance of specific safeguard sy__em designs, and to discuss some important measures presently used in the Lawrence Livermore Laboratory safeguards program. As a central backdrop, we use the system decomposition suggested in Ref. 1 and repeated here in Figs. 3-1 and 3-2.

In Fig. 3-1, a safeguard system is viewed as a three-level hierarchical decision structure where subsystemis at one 'evel constrain or control subsystems at lower levels. In Fig. 3-2, the physical process subsystem at the lowest level is further decomposed into three basic elements: people management, material control, and material accounting. Each of these has three subelements: measurement, de $\therefore^{-} \cdot \mathrm{n}$, and action.

Consider Fig. 3-3, which is an aggregation of Fig. 3-2 into two basic building blocks: the detection subsystem $(D)$, and the response subsystem $(R)$. In that model, $\theta_{D}$ and $\theta_{R}$ are structural variables or processes representing the nature of tampering and other structural events discussed in detail in Ref. 1. The variables $\omega_{D}$ and $\omega_{R}$ represent the multidimensional states and environmental conditions of subsystems $D$ and $R$, respectively. These can be used, for instance, to describe system operating modes, uncertainties in system properties, external disturbances, the failure conditions or states of system components, and so on.

The various variables in Fig. 3-3 should be visualized as sequences of acts, detection signals, responses, or states. A sequence is an ordered collection of elements. These elements are the terms of the sequence." When only the terms are of interest, but the ordering is not, these terms simply constitute a set. Although the words "set" and "event set" occasionally stand for both a set of things and a sequence of things in the SAA and SVAM methodologies at LLL, we shall be careful to distinguish a set of real numbers from an ordered set of real numbers, or sequence of real numbers.

In Fig. 3-3, an adversary is simply represented by a sequence of nontampering acts (inputs) $u=\left(u_{1}, u_{2}, \ldots, u_{n}\right)$ from an input space $U$, detection-subsystem tampering acts $\theta_{D}=\left(\theta_{D 1}, \theta_{D 2}\right.$, $\left.\ldots, \theta_{D m}\right)$, and response-subsystem tampering acts $\theta_{R}=\left(\theta_{R 1}, \ldots, \theta_{R p}\right)$, from parameter spaces $\mathrm{e}_{\text {? }}$ ? and $\Theta_{R}$, respectively. For this report, an adversary is thus represented by a triple $\left(u, \theta_{D}, \theta_{R}\right)$. it should be easy to see that adversary resource levels, frequencies of attempts, goal quantities, and other generic adversary descriptions should ultimately be expressed in such a form when detailed evaluation or assessment models are to be exercised. Note that we specialize our unput space $U$ to describe adversary action sequences only. It is easy to see that $U$ can also include "legitimate" acts, and the purpose of the detection subsystem $D$ must then be broadened to separate legitimate from illegitimate acts. This would require the study of false alarm conditions, which we do not consider explicitly in this report.

In addition to the input space $U$ and the parameter spaces $\Theta_{D}$ and $\Theta_{R}$, we also have state spaces $\Omega_{D}$ and $\Omega_{R}$ whose members are the states $\omega_{D}$ and $\omega_{R}$, and output spaces $V$ and $W$, whose members are detection outputs $v$ and response outputs (responses) $w$, respectively.

As we said earlier, if a collection of things (acts, states, ...) is of interest but their ordering is not, then it may be expressed simply as a set. For instance, if the order in which an adversary commits nontampering acts is irrelevant, then this collection of acts may be represented as the set $u=\left\{u_{1}, \ldots, u_{n}\right\}$. Otherwise, it will be represented as a sequence $u=\left(u_{1}, u_{2}, \ldots, u_{n}\right)$. (Note the difference in the brackets.)

\footnotetext{
-Formally, a sequence $s$ is a function from a subset $N^{\prime}$ of the positive integers $N$ which preserves the natural ordering of the integers. For the value of the sequenr $: s$ at integer $i$, we write $s_{i}$, rather than $s(i)$. If $N^{\prime}$ is countable, then $s$ is countable, and we represent $s$ by $\left(s_{1}, s_{2}, \ldots\right)$. If $N^{\prime}$ is finite, $s$ is finite, and we write $s=\left(s_{1}, s_{2}, \ldots, s_{n}\right)$, where
$n \in N$.
} 


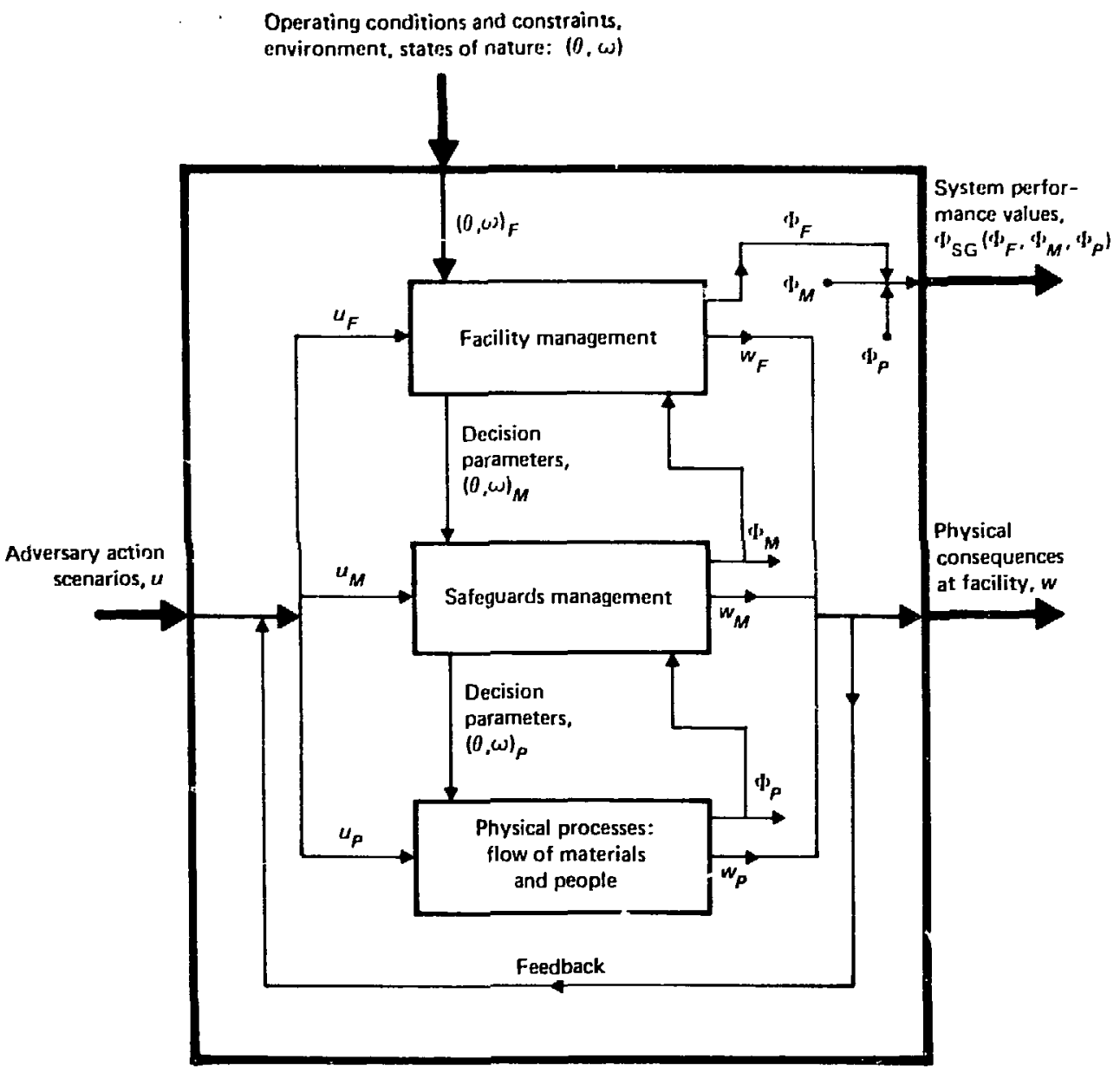

Fig. 3-1. The decision hicrarchy in a gencral safeguard system SG whose performance $\Phi_{S G}$ is a function of subsystem performances $\Phi_{F}, \Phi_{M}$, and $\Phi_{P}$.

Now that we have defined the external attributes of our subsystems, let us establish their internal structure. The detection subsystem $D$ is a function $D: U \times \Omega_{D} \times \Theta_{D} \rightarrow V$, where $U \times \Omega_{D}$ $\mathrm{X} \Theta_{D}$ is the product of the input (action) set $U$, detection subsystem state set $\Omega_{D}$, and tampering set $\Theta_{D}$. In response to an input sequence $u \in U$ and tampering sequence $\theta_{D} \in \Theta_{D}$, and for a state $\omega_{D} \in \Omega_{D}$, the detector subsystem $D$ produces an output $D\left(u, \omega_{D}, \theta_{D}\right)=v=\left(\left(u_{1}, d_{1}\right), \ldots,\left(u_{n}, d_{n}\right)\right)$ $\in V$, where each pair $\left(u_{i}, d_{i}\right)$ is a nontampering act and the corresponding detection signal, and $\left(d_{1}\right.$, $d_{2}, \ldots, d_{n}$ ) is the detection signal sequence. Such pairs may obviously be used to describe a wide variety of detector outputs, and might denote events, probabilities, distributions, and so on. For instance, $u_{i}$ might be "the adversary steps on floormat $F$," and $\left(u_{i}, d_{i}\right)$ might be "adversary stepping on $F$ produces a detection signal $d_{i}$," or "adversary stepping on $F$ is detected," or various interpretations suitable to the modeler. If specific information concerning the detection of tampering acts 


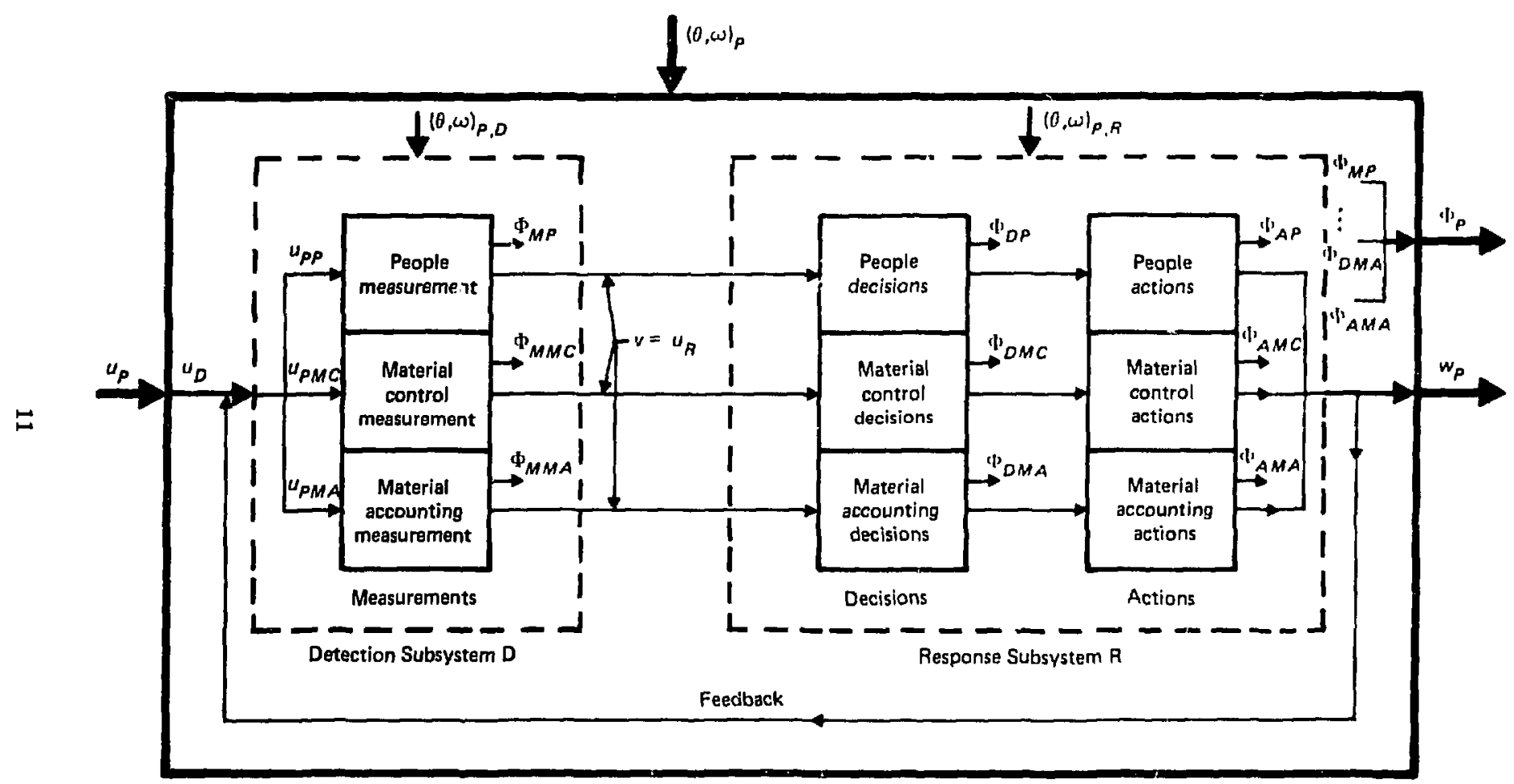

Fig. 3-2. The physical process level of Fig. 3-1 decomposed into its two major subsystems: the detection subsystem $\dot{D}$, and the response subsystem $R$, which themselves consist of three and six subsystems, respectively. 


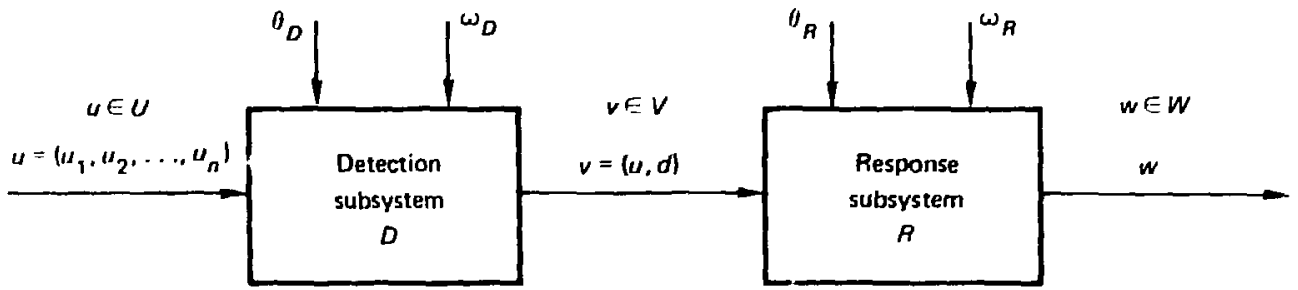

Adversary Action

Action-Detection

Responses

Sequences $u$

Pairs $(u, d)$

Fig. 3-3. An aggregated model of a safeguard system. Inputs are adversary action $u$, tampering acts $\left(\theta_{D}, \theta_{R}\right)$, and plant state $\left(\omega_{D}, \omega_{R}\right)$. Outputs arc detection outputs $v$ and diversion outcomes $w$.

is requircd in the detection output $v$, it can be included by simply adding values of the variable $\theta_{D}$ whenever needed. A component $v_{i}$ of $v$ would then be a triple $\left(u_{i}, \theta_{d_{i}}, d_{i}\right)$, but this would make the present analysis unnecessarily cumbersome, and hence we have omitted it. This modeling approximation is not as serious as it may seem at first, because tampering acts $\theta_{D}$ are intimately associated with adversary movements throughout the plant, or with other acts which are nontampering inputs $u$ to the system. While the detection of a tampering act $0_{D}$ is not explicitly represented in our initial description of $v$, any detection events associated with acts $u$ surrounding such a tampering act could be included as $\left(u_{i}, d_{i}\right)$ pairs if necessary.

The response system $R$ is a function $R: V \times \Omega_{R} \times \Theta_{R} \rightarrow W$. It operates on detection outputs $v \in V$ and responds to such outputs, tampering acts $\theta_{R}=\left(\theta_{R 1}, \ldots, \theta_{R p}\right)$, and response system states $\omega_{R}=\left(\omega_{R 1}, \ldots, \omega_{R p}\right)$ by producing an output $R\left(v, \omega_{R}, \theta_{R}\right)=w \in W$. This output could also be probabilistic or deterministic, just as for the detection subsystem. The space of outputs $W$ might consist of just two points or events: $w_{1}$ ("apprehension of adversary with any material taken"), and $w_{2}$ ("nonapprehension," or successful diversion). Or the variable $w$ might be the probability that the adversary is "suitably apprehended." In the model of Fig. 3-3, therefore, an adversary committing sequences of acts $u, \theta_{D}$, and $\theta_{R}$ against a facility in state $\left(\omega_{D}, \omega_{R}\right)$ produces a detection output $D\left(u, \omega_{D}, \theta_{D}\right)=v=\left(\left(u_{1}, d_{1}\right), \ldots,\left(u_{n}, d_{n}\right)\right)$, and an overall system response $w=R\left(D\left(u, \omega_{D}, \theta_{D}\right), \omega_{R}, \theta_{R}\right)$.

In the Appendix, we formally represent this overall system as a structured system in the framework developed in Ref. 1. Throughout this paper, we shall use commonplace set operarions like union, intersection, and complementation, and it might be well now to review some notation. When an indexed or parametrized collection of sets $\left\{A_{i}: i \in I\right\}$ or $\{A(i): i \in I\}$ is considered, we shall denote the intersection and union of the elements of this collection by $\bigcap_{\mathrm{j} \in I} A_{i}$ and $\bigcup_{i \in J} A_{i}$, respectively, or by $\bigcap_{i \in I} A(i)$ and $\bigcup_{i \in I} A(i)$, respectively. We denote the complement of a set $A$ by $\mathscr{C}(A)$, the difference between $A$ and $B$ relative to $A$ by $A-B=A \cap \mathscr{C}(B)$, and the symmetric difference $(A-B) \cup(B-A)$ by $A \Delta B$. Another convention we shall use applies to functions of several variables when some of these are held fixed. Considering the detection function (nontampering case) $D: U \times \Omega_{D} \rightarrow V$, we use the symbol $D_{u}$ to denote the function from $\Omega_{D}$ to $V$ obtained by fixing the first argument $u$, and the symbol $D_{u}^{-1}(v)$ or $\Omega_{u}(v)$ to indicate the set $\left\{\omega \in \Omega_{D}\right.$ : $D(u, \omega)=v \in V, u$ fixed $\}$, and similarly for $D_{\omega}, D_{\omega}^{-1}(v)$. 


\section{PROBABILISTIC ANALYSIS OF SAFEgUARD SYSTEMS}

The preceding chapter has addressed the conditional question "if $\left(\omega_{D}, \omega_{R}\right)$ is the truc state of nature for $D$ and $R$, if $\left(\theta_{D}, \theta_{R}\right)$ are the tampering acts against $D$ and $R$, and if $u$ is the adversary nontampering action sequence, does diversion occur?" The underlying assumption, of course, has been that these conditions and the associated diversion statement fully describe the diversion process without any uncertainty. In this chapter, we admit that these conditions are rarely. if ever, known with certainty and precision, and we assume that quantitative probability theory is an appropriate framework for describing our ignorance about them. We do not, therefore, consider other frameworks like fuzzy set theory, qualitative probability theories, and various other machineries presently under development.

For simplicity of exposition, but without loss of generality, we only consider the detection subsystem $D$ at this point, without tampering. Referring to Fig. 4-1, and using language developed in previous chapters, we note that $D$ is a function $D: U \times \Omega \rightarrow V$. For this system, $\omega$ is the state variable representing environmental conditions, failure states (see Ref. 4, p. 2), and operating modes of $D$, and $U$ is the usual space of action sequences. Recalling some mathematical conventions, we shall use the symbol $D_{u}$ to represent the function from $\Omega$ to $V$ obtained from $D$ by fixing the first argument $u$, the symbol $D_{u}^{-1}(v)$ or $\Omega_{u}(v)$ to denote the set $\{\omega \in \Omega: D(u, \omega)=v \in V, u$ fixed $\}$, and similarly for $D_{\omega}, D_{\omega}^{-1}(v)$, and $U_{\omega}(v)$.

The principal difference between our present approach and eurlier work is that we now consider both the input sequences $u$ and states $\omega$ as probabilistic objects. For nontrivial $D$ functions, therefore, the detection output $v=D(u, \omega)$ is also uncertain and may be informally described as a "random variable."

It has been observed that the terms randomness, uncerrainty, probability, and random variables connote very different things to different people, and therefore in the following section we present a brief review of some elementary concepts that will play an important role in our analysis. For those readers who find this summary of introductory probability insufficient, a thorough treatment can be found in the basic works of Breiman ${ }^{5}$ and Chung. ${ }^{6}$

\subsection{REVIEW OF SOME ELEMENTARY CONCEPTS IN PROBABILITY THEORY}

Probability theory is a framework used to describe and analyze experiments whose outcomes are uncertain. Such experiments need not be anything even remotely resembling a laboratory experiment. They can be purely abstract experiments, gedanken experiments, computer experiments, and so on.

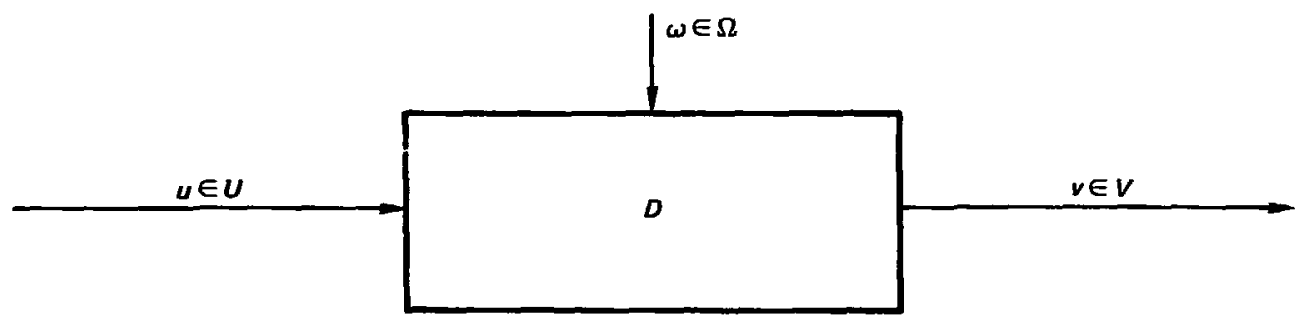

Fig. 4-1. A simple detection subsystem representation with input space $U$ (action space), plant state space $\Omega$, and output space $V$ (diversion outcome space). 
The most basic element of an experiment is the set of possible or conceivable outcomes of the experiment. This is called the sample space of the experiment, and we shall refer to it as $\Omega$. In the throwing of a die, for instance, the possible outcomes would be the six faces of the dic, identified by the set of numbers $\{1,2,3,4,5,6\}$. Note that this is a modeling statement of sorts, since we reject the possibility that the die comes to rest on one of its edges, or even gets lost.

The second basic element of an experiment is a collection of combinations of outcomes of interest to the experimenter. Each combination of outcomes is a subset $A$ of $\Omega$, called an event, and we refer to the collection of these cvents in an experiment as $|a|$, the event set. The size of this collection determines the degree of detail with which the experiment is modeled. For fine detail, all subsets of $\Omega$ (i.e., the power set of $\Omega$ ) may be included in $|a|$, and for a course analysis, just a few events may be of interest, as in the die example below. In order to allow the discussion of disjuncive, conjunctive, and negation statements, the set $|\alpha|$ is endowed with the basic set-theoretic operations of union, intersection (countable or finite), and complementation. When the set $|\alpha|$, endowed with these operations and including the empty set $\theta$ and the sample space $\Omega$, is closed under the operations, it constitutes a o-algebra of events, which we denote by $a$ and refer to as the event space. Using our example of a die, the experimenter may be interested in just those events where the outcome of the experiment of throwing the die results in an even number. In this case, his event space $a$ would be $\{0,\{2,4,6\},\{1,3,5\}, \Omega\}$ where $\emptyset$ is the null event and $\Omega$ is the sure event Note that the event $\{1,3,5\}$ must be included because the space $a$ must be closed under the cumplementation operation $\mathscr{B}$, and $\mathscr{B}(\{2,4,6\})=1,3,5 \subset \Omega$.

The next basic element of an experiment is a rule $P$ which associates with each event $A \in a$ a number $P(A) \in[0,1]$, the probability of $A$. This rule represents the experimenter's information, belief, or even faith about the likelihood of pccurrence of any of the outcomes in $A \in a$. For instance, if the die is "fair," and all outcomes are equiprobable, $P(\{2,4,6\})=0.5$.

These three elements together constitute the formal concept of an experiment. Thus, an experiment is a triple $\mathscr{E}=\langle\Omega, a, P\rangle$, where

$\Omega$ is the space of outcomes or samples,

$a$ is the space of events (a $\sigma$-algebra),

$P: a \rightarrow[0,1]$ is the probability measure, or distribution of $\mathscr{E}$.

An experimenter is rarely interested in all the events or outcomes of an experiment. He will often observe the results of an ongoing experiment (nature, for instance) by means of measuring instruments and computation devices. These usually provide aggregated information of more value to the experimenter than all the "raw" data. To characterize this aggregation process, we must introduce another fundamental idea in probability theory: the random variable. Starting with an

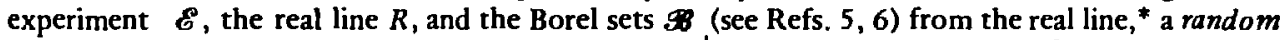
variable is a function $X: \Omega \rightarrow R$ which is measurable ${ }^{\dagger}$ with respect to $a$,i.e., $X^{-1}(\mathscr{B}) \subset a$. Thus, the inverse $X^{-1}$ of $X$ maps every Borel set $B \in \mathscr{B}$ to an event $X^{-1}(B)=A \in Q$. This measurability condition is important because, without it, we cannot establish the probability of all the intervals on the real line that may be important to us, because some of these intervals will not have a corresponding event in $\mathbf{a}$. Because $P$ is the original probability and is defined on $a$ only, $P\left(X^{-1}(B)\right)$ is not defined for such intervals $B \in \mathscr{B}$.

The distribution function $P_{X}$ of $X$ is the probability measure induced on the Borel sets by $X$ and $P$ as follows. For any Borel $\operatorname{set}^{* *} B \in \mathscr{B}, P_{X}(B)=P\left(X^{-1}(B)\right)$. Since $X$ is measurable, these quantities are well defined.

The cumulative distribution function $F_{X}$ of $X$ is the real-valued function on the codomain of $X$ defined by

\footnotetext{
Roughly speaking, these are all the intervals on the real line.

${ }^{\dagger}$ Also called $a$-measurable.

* "We shall also express $P_{X}(B)$ in the form $P(\{\omega: X(\omega) \in B\})$ whenever the explicit dependence of values of $X$ on $\omega$ is needed.
} 


$$
F_{X^{x}}(x)=P_{X^{(}}((-\infty, x))=P\left(X^{-1}(-\infty, x]\right) .
$$

Again, since $X$ is measurable, $F_{X}(x)$ is well defined. We shall also consider the extension of a random variable to the multidimensional case, ${ }^{5,6}$ where we have a vector-valued random variable $X: \Omega \rightarrow R^{n}$, the $n$-dimensional Euclidean space of reals (product space) with the $n$-dimensional Borel sets $\boldsymbol{B}^{n}$. When $x$ is a vector of sealars $\left(x_{1}, x_{2}, \ldots, x_{n}\right)$, we denote by $(-\infty, x]$ the product subset

$$
\prod_{i=1}^{n}\left(-\infty, x_{i}\right] \subset R^{n}
$$

Finally, if $F_{X}$ has a (Radon-Nikodym) derivative* (Refs. 5,6 ) with respect to the Lebesgue measure on $\mathscr{B}$, we denote it by $f_{X}$ and call it the density function of $X$.

To conclude our die example, we consider the function $X: \Omega \rightarrow R$ which assigns to each even face the value 1 and to each odd face the value 0 . It is easy to show that $X$ is measurable, and that

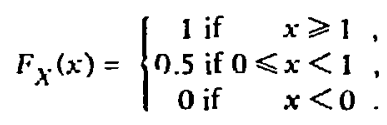

Obviously, $f_{X}$ does not exist for this random variable.

\subsection{THE DETECTION SUBSYSTEM AS A PARAMETRIZED COLLECTION OF RANDOM YARIABLES}

Returning to Fig. 4-1, we note that if both the adversary actions $u$ and state $\omega$ are treated as uncertain variables whose values are determined by conditions outside the scope of $D$ (external or exogenous conditions), then these may be naturally considered as outcomes of some associated experiment

$$
\mathscr{E}=\langle U \times \Omega, \mathscr{U} \times a, P\rangle,
$$

where $\mathscr{Q} \times \mathrm{X}$ is a product $\sigma$-algebra of pairs of events $\left(A_{U}, A_{\Omega}\right) \subset U \times \Omega$, and $P$ is a probability on $\mathscr{Q} \times a$.

If $V$, the $2 n$-dimensional space of real-valued sequences $(u, d)$, is endowed with some detection event space $\mathcal{W}$, and $D: U \times \Omega \rightarrow V$ such that $D^{-1}(\mathscr{V}) \subset \mathscr{Q} \times a$, then the detection subsystem $D$ is simply a vector-valued random variable from $U \times \Omega$ into $V$.

We can now express our fundamental modeling philosophy a bit more intuitively. While it makes sense to ask "what is the probability that a given action sequence $u$ be detected?" it does not make sense to ask "if the action sequence is $u$, and the system state is $\omega$, what is the probability that $u$ be detected?" The reason is that we have externalized all system uncertainties in terms of $\Omega$ and the experiment (probability space) $\mathscr{E}_{\Omega}=\langle\Omega, a, P\rangle$. Any relevant source of uncertainty related to detection is captured in $\mathscr{E}_{\Omega}$. If both $u$ and $\omega$ are known; then either $D$ detects $u$ or not, for sure. Thus, $D: U \times \Omega \rightarrow V$ is a deterministic structure which may accept stochastic inputs, thereby producing stochastic outputs, but these outputs are produced in a deterministic ("entirely predictable") manner. The same approach will be used in modeling the response subsystem and the safeguard system as a whole.

"If things are "nice," this is simply the ordinary derivative of $F_{X}(x)$ with respect to $x$. 
Probabilities of detection outputs can now be computed in the obvious way. If every actiondetection sequence pair $(u, d)$ is an event in $\mathscr{W}$, then the probability of occurrence of such an actiondetection pair is $P_{D}(u, d)=P\left(D^{-1}(u, d)\right)=P\left(u, \Omega_{u}\right)$, where $\Omega_{u}=\{\omega \in \Omega: D(u, u)=(u, d)\}$ Since $P$ is a joint probability on $U$ and $a$, and the events $u$ and $\Omega_{u}$ are not necessarily independent, then $P\left(u, \Omega_{u}\right)=P_{U}(u) P\left(\Omega_{u} / u\right)$, where $P\left(\Omega_{u} / u\right)$ is the conditional probability of $\Omega_{u}$ given action $u$. More informally, $\Omega_{u}$ is the set of system states that, together with fixed input $u_{\text {, produce a detec- }}$ tion sequence $d$. Since the action $u$ chosen by some adversary may depend on the state $\omega, u$ and $\Omega_{u}$ are not independent. If actions are independent of states, then $P$ may be decomposed into its two marginal components $P=\left(P_{4}, P_{\Omega}\right)$, one for the action event space $\mathscr{Q}$, the other for the state event space $a$.

Let us now turn to the case where we fix $u \in U$, and want to determine the probability that a given detection sequence $d$ be produced by that $u$. This is equivalent to asking: "what is the conditional probability $P_{V}(d / u)$ of $d$ given $u$ ?" Because the conditional distributions $P_{V}(d / u)$ are random variables ${ }^{5,6}$ on the space or experiment $\left\langle U, \mathscr{U}, P_{U}\right\rangle$, and we are not interested in the probability $P_{U}$, we need not carry this whole experiment with us, and it is better to consider the parametrized collection $D_{U}=\left\{D_{u}: u \in U\right\}$ consisting of random variables $D_{u}: \Omega \rightarrow V$, one for each $u \in U$. It is now an easier matter to determine the probability that a fixed $u \in U$ produces a given detection sequence $d$. It is simply $P_{\Omega}\left(\Omega_{u}(v)\right)$, where $\Omega_{u}(v)=D_{u}^{-1}(v)$, and $v=(u, d)$. We now also have a collection of cumulative distribution functions $\boldsymbol{F}_{D_{U}}=\left\{F_{D_{u}}: u \in U\right\}$, where for each $u \in U$, $F_{D_{u}}(v)=P_{D_{u}}((-\infty, v])=P_{\Omega}\left(D_{u}^{-1}((-\infty, v])\right)$, a cumulative distribution ${ }^{*}$ on $V$.

Notice that $v$ is a $2 \pi$-dimensional vector $\left(u_{1}, \ldots, u_{n}, d_{1}, \ldots, d_{n}\right)$, and by $(-\infty, v]$ we mean the product subset ${\underset{X}{i}=1}_{1}^{2 n}\left(-\infty, \nabla_{i} l \subset R^{2 n}\right.$. 


\section{SOME IMPORTANT SAFEGUARD QUESTIONS}

Now that we have the mathematical machinery for addressing safeguard questions precisely. let us inquire how some specific questions are expressed in this framework. First we discuss deterministic questions, then broaden our discussion to probabilistic questions, then consider the general case of tampering.

\subsection{DETERMINISTIC QUESTIONS}

In this section, we discuss some of the deterministic questions that models developed at LLL have been designed to answer. First, we deal with nontampering adversaries, then with the general case of tampering adversaries.

\subsubsection{Nontampering Adversaries}

For this category, the spaces $\Theta_{D}$ and $\Theta_{R}$ are not included; the detection subsystem is a funcrion $D: U \times \Omega_{D} \rightarrow V$, and the response subsystem is a function $R: V \times \Omega_{R} \rightarrow W$. We assume throughout this section that only two responses are of interest: successful diversion $\left(w_{2}\right)$, and unsuccessful diversion $\left(w_{1}\right)$. This is obviously only a rough description of system response, but a more detailed and accurate description of the response variable $w$ will not significantly affect our analysis, and will only make it more complex. Our output space $W$ is thus simply the pair $W=\left\{w_{1}, w_{2}\right\}$.

1. What Are the Sequences of Acts $u=\left(u_{1}, u_{2}, \ldots, u_{n}\right)$ that Lead to Successful Diversion, $w=w_{2}$ ? It is clear that the success of a sequence $u$ in producing diversion $\left(w=w_{2}\right)$ depends on the plant state $\left(\omega_{D}, \omega_{R}\right)$. The pairs $\left(v, \omega_{R}\right)$ of detection outputs $v$ and response subsystem states $(R$ states) that produce $w_{2}$ are $R^{-1}\left(w_{2}\right)=\left\{\left(v, \omega_{R}\right) \in V \times \Omega_{R}: R\left(v, \omega_{R}\right)=w_{2}\right\} \subset V \times \Omega_{R}$. The combinations of nontampering inputs $u$ and plant states $\left(\omega_{D}, \omega_{R}\right)$ that produce successful diversion $w_{2}$ are thus

$$
\operatorname{Div}=\left\{\left(u, \omega_{D}, \omega_{R}\right) \in U \times \Omega_{D} \times \Omega_{R}:\left(D\left(u, \omega_{D}\right), \omega_{R}\right) \in R^{-1}\left(w_{2}\right)\right\}
$$

If the plant state is $\left(\omega_{D}, \omega_{R}\right)$ and $\left(u, \omega_{D}, \omega_{R}\right) \in \operatorname{Div}$, then $u$ is a successful diversion action sequence (produces $w_{2}$ ).

The vulnerability of a safeguards system depends on the state of the system, and many relevant questions can be asked with respect to plant states $\left(\omega_{D}, \omega_{R}\right)$. We address just one here, and will present several important ones when knowledgeable or informed adversaries are discussed.

2. What Are the Sequences of Acts $u$ that Lead to Successful Diversion Regardless of Plant State $\left(\omega_{D}, \omega_{R}\right)$ ? The listing of all input, plant-state combinations $\left(u, \omega_{D}, \omega_{R}\right)$ that cause diversion can be a complex matter. The measure discussed here eliminates the need to carry along all the states $\left(\omega_{D}, \omega_{R}\right)$ for which diversion can take place. This measure is obviously only a rough approximation to all the interesting inputs since it does not account for those inputs which may "work" for some states and not for others. Any safeguard system possessing such sequences is obviously a very poor system, and this measure provides a good test for an important minimal requirement.

We are interested in those sequences which will produce $w_{2}$ (diversion) for all plant states (e.g., failure states) $\left(\omega_{D}, \omega_{R}\right) \in \Omega_{D} \times \Omega_{R}$, where $\omega_{D}$ is the detection subsystem state and $\omega_{R}$ is the response subsystem state. The detection outputs (response system inputs) that produce a $w_{2}$ for all response subsystem states $\omega_{R}$ are

$$
V_{*}=\bigcap_{\omega_{R} \in \Omega_{R}}\left\{v:\left(v, \omega_{R}\right) \in R^{-1}\left(w_{2}\right)\right\},
$$


where $\left\{v:\left(v, \omega_{R}\right) \in R^{-1}\left(w_{2}\right)\right]$ is the set $A\left(\omega_{R}\right)$ of detection outputs associated with diversion when the $R$-state is $\omega_{R}$. Similarly, the set of inputs $u$ that lead to successful diversion, regardless of plant state $\left(\omega_{D}, \omega_{R}\right)$, is

$$
U_{*}=\bigcap_{\omega_{D} \in \Omega_{D}}\left\{u: D\left(u, \omega_{p}\right) \in V_{*}\right\} .
$$

There are several questions of particular interest when the adversary is assumed to possess special resources and privileged information concerning the state $\left(\omega_{D}, \omega_{R}\right)$ of the plant at any time. He could then wait, for instance, until the plant is in a certain state and commit those acts which will guarantec success. We address two deterministic questions next.

3. What Are the Sequences of Acts $u$ that Lead to Successful Diversion for at Least One Plant State $\left(\omega_{D}, \omega_{R}\right)$ ? The success of an attempt clearly depends on the state of the plant. Here we are interested in finding those combinations of acts which produce a diversion $w_{2}$ for at least one plant state $\left(\omega_{D}, \omega_{R}\right)$. The pairs $\left(v, \omega_{R}\right)$ of detection outputs $v$ and response subsystem states $\omega_{R}$ that produce $w_{2}$ are clearly $R^{-1}\left(w_{2}\right)$. We do not care which $R$-state $\omega_{R}$ is associated with successful diversion $w_{2}$, but only which detection outputs $v$ produce $w_{2}$ for at least one $\omega_{R}$. This set is

$$
V^{*}=\bigcup_{\omega_{R} \in \Omega_{R}}\left\{v \in V:\left(v, \omega_{R}\right) \in R^{-1}\left(w_{2}\right)\right\},
$$

the union of all detection outputs $v$ that are associated with successful diversion, regardless of $R$-state $\omega_{R}$.

Next, the set of adversary act sequences and $D$-state pairs $\left(u, \omega_{D}\right)$ that produce points in $V^{*}$ is clearly $D^{-1}\left(V^{*}\right)$, where $V^{*}$ is the set of detection outputs defined above. The set of act sequences that produce successful diversion for at least one plant state $\left(\omega_{D}, \omega_{R}\right)$ is thus

$$
U^{*}=\bigcup_{\omega_{D} \in \Omega_{D}}\left\{u \in U:\left(u, \omega_{D}\right) \in D^{-1}\left(V^{*}\right)\right\} .
$$

In this paper, we call this the set of successful actions by knowledgeable adversaries.

Whereas the previous question deals with weaknesses of the system to adversary action inputs, there is a natural converse of this question which inquires about the states in which the system is vulnerable to some input.

4. What Are the Plant States $\left(\omega_{D}, \omega_{R}\right)$ for Which at Least One Input Sequence $u$ Causes Diversion? When data about adversary types are scant, or it is difficult to assess the impaci of particular adversary strategies (the $\theta$ and $u$ sequences, for instance) upon the safeguard system, it is desirable to have some measure of system vulnerability or performance that does not require any information about $\theta$ and $u$. One way to develop such a measure is to look at all the plant states (system and component operating conditions, for instance) for which at least one adversary strategy $u$ leads to diversion. This set, which is a basic output of the SVA method (see Chapter 7) is described here. Another way to interpret this set is in terms of another type of knowledgeable and resourceful adversary: the adversary who knows when the system is in one of many states $\Omega_{D}^{\prime} \times \Omega_{R}^{\prime} \subset \Omega_{D} \times \Omega_{R}$, knows the appropriate action $u$ such that $\left(u, \omega_{D}, \omega_{R}\right)$ leads to diversion, and can implement such a $u$ after waiting for the system to be in one such state.* If there is at least one such $u$, then the state $\left(\omega_{D}, \omega_{R}\right)$ associated with that $u$ qualifies as a member of the set we seek. This set is simply

$$
\Omega^{*} \stackrel{\mathrm{DEF}}{=} \bigcup_{u \in U} A_{\mathbf{u}}
$$

Tampering is not specifically considered here. 
where

$$
A_{u} \stackrel{\text { DEF }}{=}\left\{\left(\omega_{D}, \omega_{R}\right): R\left(D\left(u, \omega_{D}\right), \omega_{R}\right)=w_{2}\right\}
$$

We thus have two ways to analyze an informed and resourceful adversary: by listing the states in which the facility is vulnerable to such an adversary, or by listing the input sequences to which the facility is vulnerable for at least one plant state. This information can be represented by an undirected bipartite graph $G=\langle N, E\rangle$ (Fig. 5-1) whose nodes or vertices are $N=U \cup \Omega_{D} \times \Omega_{R}$, and where $E \subset U \times\left(\Omega_{D} \times \Omega_{R}\right)$ are the edges between nodes in $U$ and nodes in $\Omega_{D} \times \Omega_{R}$. We shall denote by $E\left(u_{i}\right), i=1,2, \ldots, n$, the states $\omega \in \Omega_{D} \times \Omega_{R}$ related to $u_{i}$ by an edge, and by $E^{-1}\left(\omega_{j}\right)$, $j=1,2, \ldots, n$, the inputs $u \in U$ related to $\omega_{j}$ by an edge. There is an edge between $u_{k} \in U$ and $\omega_{j} \in \Omega_{D} \times \Omega_{R}$ iff input $u_{k}$, together with state $\omega_{j}$, causes a diversion. Thus, edges of $G$ represent $\left(u_{i}, \omega_{k}\right)$ pairs that lead to diversion. We call the nodes in such pairs critical nodes. We also denote by $d(t)$ the degree of node $t \in N$ (the number of nodes related to $t$ by an edge).

If a resourceful adversary such as the one above intends to perpetrate a particular $u_{i}$ with degree $d\left(u_{i}\right)>0$, thersfore, he will wait until the system is in one of the staies in $E\left(u_{i}\right)$. We do not discuss here the length of time he may have to wait, or how long a system is in a given state. Conversely, if he knows the system is in state $\omega_{j}$, he may perpetrate one of the acts $u \in E^{-1}\left(\omega_{j}\right)$. For this adversary, therefore, system hardness ; ayy be analyzed either in terns of critical states or critical inputs alone. If the system is in any of the states $\left\{\omega \in \Omega_{D} \times \Omega_{2},: d(\omega)>0\right\}$, or if any act $u_{i} \in\{u \in U: d(u)>0\}$ is attempted, diversion may be assumed to occur. Therefore, the system may be hardened either by preventing its being in any such state or by protecting against any such input, although in practical and complex systems, a combination of both schemes would always be present to some extent. For an initial analysis, one may wish to decide whether to concentrate on states or inputs, and one could use the degree or valence of all the nodes as a criterion. If the combined tegree of $\Omega_{D} \times \Omega_{R}$ nodes considerably exceeds the combined degree of $U$-nodes, ${ }^{*}$ one might prefer to analyze the states since there might be "fewer" critical states than critical inputs. This could be an important computational consideration. Of course, one must take into account that

$$
\begin{aligned}
& d\left(u_{8}\right)=1 \\
& d\left(u_{7}\right)=2 \\
& d\left(u_{6}\right)=1 \\
& d\left(u_{5}\right)=1 \\
& d\left(u_{4}\right)=3 \\
& d\left(u_{3}\right)=1 \\
& d\left(u_{2}\right)=1 \\
& d\left(u_{1}\right)=0
\end{aligned}
$$

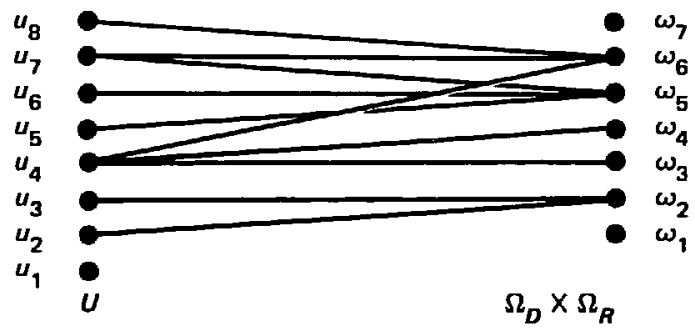

$$
\begin{aligned}
& d\left(\omega_{7}\right)=0 \\
& d\left(\omega_{6}\right)=3 \\
& d\left(\omega_{5}\right)=3 \\
& d\left(\omega_{4}\right)=1 \\
& d\left(\omega_{3}\right)=1 \\
& d\left(\omega_{2}\right)=2 \\
& d\left(\omega_{1}\right)=0
\end{aligned}
$$

Fig. 5-1. Bipartite graph illustrating the input, plant-state pairs $(u, \omega)$ that give diversion and the degree of each node (i.e., number of opposite nodes it pairs with to give diversion).

\footnotetext{
"The combined degree of a subset of nodes might simply be the weighted sum of the degrees of its members.
} 
any $u_{i}$ could be related to several $\omega_{j}$ 's. For the graph of Fig. 5-1, for instance, the combined unweighted degrees of inputs and states are

$$
d(U)=\sum_{i=1}^{8} d\left(u_{i}\right)=10
$$

and

$$
d\left(\Omega_{D} \times \Omega_{R}\right)=\sum_{i=1}^{7} d\left(\omega_{i}\right)=10
$$

respectively. Since $d(U)=d\left(\Omega_{D} \times \Omega_{R}\right)$, other criteria or a weighting must be introduced to reach a decision.

When probabilistic analysis is contemplated, for instance, an additional argument in favor of analyzing states is that probabilities of states may be easier to assess than probabilities of inputs $u$. In addition, the system may have a much higher probability of being in some siates than in others.

With our decomposition of safeguard systems into two subsystems $D$ and $R$, it is important to inquire whether performance measures can be decomposed accordingly. If we partition the sequences $u$ into detected and undetected sequences, then a simple decomposition can be obtained. In Section 7.3 we show that this decomposition is additive in the sense that the performance of the system is the sum of the performances of its subsystems $D$ and $R$. The following questions indicate how this decomposition is exploited to assess subsystem performances.

5. What Are the Combinations of Undetected Sequences of Acts $u$ and Plant States $\left(\omega_{D}, \omega_{R}\right)$ that Lead to Diversion? We consider the binary detection situation where either $d_{i}=1$ (detection) or $d_{i}=0$ (no detection), for all $i=1,2, \ldots, n$. We also define a sequence $\left(u_{1}, u_{2}, \ldots, u_{n}\right)$ to be detected iff any act $u_{i}$ in the sequence is detected. A detection output $v$ containing an element $\left(u_{i}, 1\right)$ thus means that act $u_{i}$ is detected and the associated sequence is also detected. For the purposes of this analysis, then, we define a simplified detection space $V^{\prime}=U \times\{0,1\}$, and $D\left(z, \omega_{D}\right)$ $=(u, 1)$ iff $u$ is detected when the $D$-state is $\omega_{D}$, and $D\left(u, \omega_{D}\right)=(u, 0)$ iff $u$ is not detected in that state. We then obtain a constrained set of $\left(v, \omega_{R}\right)$ pairs,

$$
\left(R^{-1}\left(w_{2}\right)\right)_{d=0} \stackrel{\mathrm{DEF}}{=} R^{-1}\left(w_{2}\right) \cap\left\{\left(v, \omega_{R}\right): v=(u, 0), \omega_{R} \in \Omega_{R}\right\}
$$

and the set of successful undetected input, plant-state sequences $\left(u, \omega_{D}, \omega_{R}\right)$ is

$$
(\text { Div })_{d=0}=\left[\left(u, \omega_{D}, \omega_{R}\right) \in U \times \Omega_{D} \times \Omega_{R}:\left(D\left(u, \omega_{D}\right), \omega_{R}\right) \in\left(R^{-1}\left(w_{2}\right)\right)_{d=0}\right\} .
$$

6. What Are the Combinations of Detected Sequences of Acts $u$ and Plant States $\left(\omega_{D}, \omega_{R}\right)$ that Lead to Diversion? This question is similar to 5 above, so we have

$$
\left(R^{-1}\left(w_{2}\right)\right)_{d=1} \stackrel{\text { DEF }}{=} R^{-1}\left(w_{2}\right) \cap\left\{\left(u, \omega_{R}\right): v=(u, 1), \omega_{R} \in \Omega_{R}\right\}
$$

and the set of successful detected input, plant-state sequences $\left(u, \omega_{D}, \omega_{R}\right)$ is

$$
(\text { Div })_{d^{\prime}=1}=\left\{\left(u, \omega_{D}, \omega_{R}\right) \in U \times \Omega_{D} \times \Omega_{R}:\left(D\left(u, \omega_{D}\right), \omega_{R}\right) \in\left(R^{-1}\left(w_{2}\right)\right)_{d=1}\right\} .
$$


Clearly, $\left(\text { Div) } d_{d=0} \cup(\text { Div) })_{d=1}=\text { Div and (Div) } d_{d=0} \cap(\text { Div })_{d=1}=0 \text {. Hence, }(\Gamma i v)_{d=0} \text { and (Div) }\right)_{d=1}$ constitute a partition of Div.

7. What Are the Undetected Sequences of Acts that Lead to Diversion for at Least One Plant State? This is similar to question 3 but with the additional no-detection constraint. We thus obtain the smaller set

$$
\left(V^{*}\right)_{d=0} \stackrel{\text { DEF }}{=} V^{*} \cap\{v=(u, 0), u \in U\},
$$

where $V^{*}$ is as defined in question 3 , and the set of successful undetected sequences for at least one plant state is

$$
\left(U^{*}\right)_{d=0}=\bigcup_{\omega_{D} \in \Omega_{D}}\left\{u \in U:\left(u, \omega_{D}\right) \in\left(D^{-1}\left(V^{*}\right)\right)_{d=0}\right\} .
$$

8. What Are the Detected Sequences that Lead to Successful Diversion for at Least One Phant State? Using the formulation of question 3, we form the set

$$
\left(V^{*}\right)_{d=1} \stackrel{\text { DEF }}{=} V^{*} \cap\{v=(u, 1), u \in U\} \text {, }
$$

and the set of successful detected sequences for at least one state $\left(\omega_{D}, \omega_{R}\right)$ is

$$
\left(U^{*}\right)_{d=1}=\bigcup_{\omega_{D} \in \Omega_{D}}\left\{u \in U:\left(u, \omega_{D}\right) \in\left(D^{-1}\left(V^{*}\right)\right)_{d=1}\right\}
$$

Clearly, $\left(V^{*}\right)_{d=0} \cup\left(V^{*}\right)_{d=1}=V^{*}$, and $\left(V^{*}\right)_{d=0} \cap\left(V^{*}\right)_{d=1}=\emptyset$. Hence, these two sets constitute a partition of $V^{*}$, a useful fact. Furthernore, it is easy to show that

$$
\begin{aligned}
\left(U^{*}\right)_{d=1} \cup\left(U^{*}\right)_{d=0}= & \bigcup_{\omega_{D} \in \Omega_{D}}\left\{u \in U:\left(u, \omega_{D}\right) \in\left(D^{-1}\left(V^{*}\right)\right\rangle_{d=1}\right\} \\
\cup & \bigcup_{\omega_{D} \in \Omega_{D}}\left\{u \in U:\left(u, \omega_{D}\right) \in\left(D^{-1}\left(V^{*}\right)\right)_{d=0}\right\}=U^{*},
\end{aligned}
$$

where $U^{*}$ is defined in question $3\left(\left(V^{*}\right)_{d=1}\right.$ and $\left(V^{*}\right)_{d=0}$ are disjoint).

9. What Are the Plant States $\left(\omega_{D}, \omega_{R}\right)$ for Which at Least One Undetected (Detected) Sequence $u$ Causes Diversion? Using the constraint of no detection, we obtain the sets

$$
B_{u} \stackrel{D E F}{=}\left\{\left(\omega_{D}, \omega_{R}\right): D\left(u, \omega_{D}\right)=(u, 0), u \text { fixed }\right\} \text {, }
$$

one for each $u \in U$. Using these together with the sets

$$
A_{u} \stackrel{\text { DEF }}{=}\left\{\left(\omega_{D}, \omega_{R}\right): R\left(D\left(u, \omega_{D}\right), \omega_{R}\right)=w_{2}, u \text { fixed }\right\},
$$

we get

$$
\left(\Omega^{*}\right)_{d=0}=\bigcup_{u \in U} A_{u} \cap B_{u} \text { (no detection) }
$$


Similarly, we get the sets

$$
C_{u} \stackrel{\text { DEF }}{=}\left\{\left(\omega_{D}, \omega_{R}\right): D\left(u, \omega_{D}\right)=(u, 1), u \text { fixed }\right\}
$$

one for each $u \in U$. With the same sets $A_{u}$ above, we obtain

$$
\left(\Omega^{*}\right)_{d=1}=\bigcup_{u \in U} A_{u} \cap C_{u} \text { (detection) }
$$

The two sets $\left(\Omega^{*}\right)_{d=0}$ and $\left(\Omega^{*}\right)_{d=1}$ are the two sets sought. Clearly, $\Omega^{*}=\left(\Omega^{*}\right)_{d=0} \cup\left(\Omega^{*}\right)_{d=1^{-}}$ These sets constitute three basic outputs of the SVA method. The extension of these sets to the case where tampering is considered is rather obvious, and we shall not discuss these further until probabilistic performarse measures are discussed in Chapter 7.

\subsubsection{Tampering Adversaries}

Adversaries who "camper" with components of a facility or safeguard system were the subject of detailed analysis in Ref. 1, where their acts were represented by the structural parameters $\theta_{D}$ and $\theta_{R}$. The detection subsystem is now a function $D: U \times \Omega_{D} \times \Theta_{D} \rightarrow V$, and the response system a function $R: V \times \Omega_{R} \times \Theta_{R} \rightarrow W$.

Because the state variables $\omega_{D}$ and $\omega_{R}$ are treated similarly to the tampering parameters $\theta_{D}$ and $\theta_{R}$ in this general setting (no specific adversaries assumed), there is no essential mathematical difference between this case and the nontampering case. We now characterize some related tampering questions in our framework.

1. What Are the Sequences of Acts $u$, System States $\left(\omega_{D}, \omega_{R}\right)$, and Tampering Acts $\theta=\left(\theta_{D}, \theta_{R}\right)$ that Lead to Successful Diversic $n$ ? The triples $\left(v, \theta_{R}, \omega_{R}\right)$ that produce $w_{2}$ (diversion) are

$$
\left(R^{-1}\left(w_{2}\right)\right)_{\mathrm{TAMP}(R)} \subset V \times \Omega_{R} \times \Theta_{R}
$$

where TAMP(R) is a subscript indicating tampering with subsystem $R$. The combinations of nontampering inputs $u$, tampering acts $\left(\theta_{D}, \theta_{R}\right)$, and plant states $\left(\omega_{D}, \omega_{R}\right)$ that produce (diversion) $w_{2}$ are thus:

$$
\begin{array}{r}
(\text { Div) })_{\mathrm{TAMP}(D, R)}=\left\{\left(u, \omega_{D}, \theta_{D}, \omega_{R}, \theta_{R}\right):\left(D\left(u, \omega_{D}, \theta_{D}\right), \omega_{R}, \theta_{R}\right)\right. \\
\left.\in\left(R^{-1}\left(w_{2}\right)\right)_{\mathrm{TAMP}(R)}\right\} .
\end{array}
$$

2. What Are the Sequence Combinations of Acts $u$ and Tampering Acts $\left(\theta_{D}, \theta_{R}\right)$ that Lead to Successful Diversion for at Least One Plant State? We first determine $R^{-1}\left(w_{2}\right) \subset V \times \Omega_{R} \times \Theta_{R}$ Since we only have cause for concern when at least one $R$-state $\omega_{R}$ and one $D$-state $\omega_{D}$ are associated with $w_{2}$, the set of detection outputs and $R$-tampering acts $\theta_{R}$ that produce diversion is

$$
A_{\mathrm{TAMP}(R)}=\bigcup_{\omega_{R} \in \Omega_{R}}\left\{\left(v, \theta_{R}\right) \in V \times \Theta_{R}:\left(v, \theta_{R}, \omega_{R}\right) \in R^{-1}\left(w_{2}\right)\right\} .
$$


Similarly, the combinations of nontampering acts $u$ and tampering acts $\left(\theta_{D}, \theta_{k}\right)$ that produce su:cessful diversion for at least one plant state are

(Div) $\left.\operatorname{TAMP}(D, R)=\bigcup_{D} \in \Omega_{D}\left\{\left(u, 0_{D}, 0_{R}\right) \in U \times \Theta_{D} \times \Theta_{R}:(I)\left(u, \omega_{D}, 0_{D}\right), 0_{R}\right) \in A_{\mathrm{TAMP}}(R)\right\}$

3. What Are the Plant States $\left(\omega_{l}, \omega_{R}\right)$ for Which at Least One Input, Tampering Triple $\left(u, \theta_{D}, \theta_{R}\right)$ Causes Diversion? $A s$ in the carlier casc, without tampering, this is the set

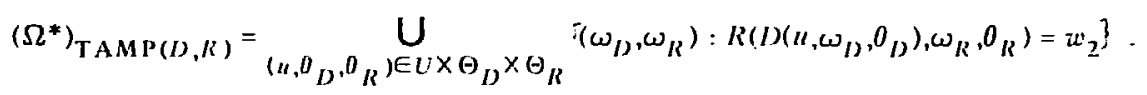

4. What Are the Undetected Sequences of Acts $\left(u, 0_{B}, \theta_{R}\right)$ that Lead to Diversion? Again, the plant state $\left(\omega_{l}, \omega_{R}\right)$ must be carried throughout. As before, we have simply

$$
\begin{aligned}
& \left(R^{-1}\left(w_{2}\right)\right)_{\operatorname{TAMP}(R), d=0}=\left(R^{-1}\left(w_{2}\right)\right)_{\operatorname{TAMP}(R)} \\
& \qquad \cap\left\{\left(v, \omega_{R}, 0_{R}\right): v=(u, 0), u \in L,\left(\omega_{R}, \theta_{R}\right) \in \Omega_{R} \times \Theta_{R}\right\} .
\end{aligned}
$$

The set of undetected sequences $\left(u, 0_{D}, 0_{R}\right)$ is

(Div) $\mathrm{TAMP}(I), R), d=0=$

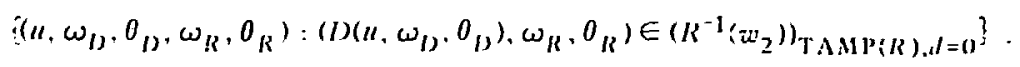

5. What Are the Detected Sequenees of Acts $\left(u, \theta_{1}, \theta_{R}\right)$ that Lead to Diversion? Similarly, the plant state $\left(\omega_{l}, \omega_{R}\right)$ must be carricd along, and we get

$(\text { Div) })_{\text {TAMP( }(I), R), t=1}=$

$$
\left.\left\{\left(u, \omega_{l}, 0_{D}, \omega_{R}, 0_{K}\right):(l)\left(n, \omega_{D}, 0_{l}\right), \omega_{R}, 0_{K}\right) \in\left(R^{-1}\left(w_{2}\right)\right)_{T \lambda M P(R), l=1}\right\},
$$

where $R^{-1}\left(w_{2}\right)$ was defined in question 3. Again, (Div) $\left.\mathrm{TAM} P(I), R\right), d=1$ and (Div) $\mathrm{TAMP}(b, R), d=0$ constitute a partition of (Div) $\mathrm{T} \wedge M \mathrm{P}(D, R)$.

Sometimes a particular plant state $\left(\omega_{D, 0}, \omega_{R, 0}\right)$ is of interest, and one may ask:

6. What Are the Sequences of Aets $\left(u, \theta_{l}, \theta_{R}\right)$ that Produce Diversion When the Plant State is $\left(\omega_{D, 0}, \omega_{R, 0}\right)$ ? Using the answers to question 1 of this section, the pairs $\left(v, \theta_{R}\right)$ that produce $w_{2}$ when $R$ is in state $\omega_{R}$ are

$$
\left.\mathrm{A}_{\mathrm{TAMP}(R), \omega_{R}=\omega_{R, 0}}=\left(v, \theta_{R}\right):\left(v, \omega_{R, 0}, \theta_{R}\right) \in R^{-1}\left(w_{2}\right)\right\} .
$$

Next, the sets of acts $\left(u, 0_{D}, 0_{R}\right)$ that produce $w_{2}$ when the facility or plant is in state $\left(\omega_{D, 0^{\prime}}\right.$ $\left.\omega_{R, 0}\right)$ are

${ }^{(D i v)} \operatorname{TAMP}(D, R),\left(\omega_{D}, \omega_{R}\right)=\left(\omega_{D, 0}, \omega_{R, 0}\right)=$

$$
\left\{\left(u, \theta_{1}, \theta_{R}\right):\left(D\left(u, \omega_{D, 0}, \theta_{D}\right), \theta_{R}\right) \in A_{\mathrm{TAMP}(R), \omega_{R}=\omega_{R, 0}}\right\}
$$


7. What Arc the Undetected Sequences of Acts $\left(1,, \theta_{l}, 0_{R}\right)$ that Lead to Successful Diversion for at Lcast Onc Plant State?

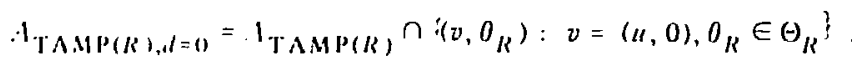

The set of suceessful undetected sequences of combinations $\left(11,0_{N}, 0_{R}\right)$ (adversaries) that lead to diversion for at least onc plant state is

$$
\left.T_{l=0}=\bigcup_{\omega_{D} \in \Omega_{l}}\left\{\left(l, 0_{l}, 0_{R}\right):(l)\left(u, \omega_{l}, 0_{l}\right), 0_{R}\right) \in A_{\mathrm{TAMP}(R), d=0}\right\}
$$

8. What Are the Detected Sequences of Acts $\left(u, \theta_{l}, \theta_{R}\right)$ that Lead to Successful Diversion for at Lcast Onc Plant State? This is similar to question 7 above, and

$$
T_{d=1}=\bigcup_{\left.\omega_{D} \in \Omega_{l}\right)}\left(\left(u, 0_{l}, 0_{R}\right):(l)\left(u, \omega_{D}, 0_{l}\right), 0_{R}\right) \in A_{\operatorname{TAMP}(R), d=l^{\prime}}
$$

As before,

$$
T_{d=0} \cup T_{d=1}=T_{,} \text {and } T_{d=0} \cap T_{d=1}=\emptyset .
$$

9. What Are the Sequences of Acts $\left(l, 0_{l}\right)$ (with No $R$-Tampering) that Lead to Diversion for at Least One Plant State? Combining previous results, we define

and

$$
V^{*}=\bigcup_{\omega_{R} \in \Omega}\left\{v \in V:\left(v, \omega_{R}\right) \in R^{-1}\left(w_{2}\right)\right\}
$$

$$
T_{D}=\bigcup_{\omega_{D} \in \Omega_{D}}\left\{\left(u, o_{D}\right):\left(u, \theta_{D}, \omega_{D}\right) \in D^{-1}\left(V^{*}\right)\right\} .
$$

These are the sequences sought.

In the next section, we enlarge the scope of our framework and address some probabilistic questions.

\subsection{PROBABILISTIC QUESTIONS}

In Section 4.2, we described the detection subsystem as a random variable $D: V \times \Omega \rightarrow V$ from the product sample space $V \times \Omega$ to the detection space $V$, and similarly for the response subsystem. If we consider fixing input sequences $u \in U$, and vary $\omega$ randomly in accordance with the probability law $P_{\Omega}$, the $D$ may be expressed as a collection of parametrized random variables $\left[D_{u}: \Omega \rightarrow V\right.$, $u \in U\}$ with $V$ as the parameter or index set.

In Section 4.1, we showed that the probability of events in the range of a random variable is determined by the inverse of the random variable and the probability of the pre-images of the events If $X$ is a random variable on a probability space $\langle\Omega, a, P\rangle$, then for any event $B$ contained in the range of $X, P_{X}(B)=P\left(X^{-1}(B)\right)$. Returning to safeguard questions, we note that the deterministic questions asked earlier are about inputs, tampering acts, and system states. These are elements of the domain of the safeguard subsystem functions $D$ and $R$, and hence are a convenient form for the determination of probabilities, because it is on the domain of $D$ and $R$ that probabilities are defined. 
Consider the question that is analogous to the fourth question of the previous section: "What is the probability that at least one input "causes diversion?" We are interested in determining the probability that the system is in state $\left(\omega_{l}, \omega_{k}\right)$ for which there is at least one action sequence " that causes diversion. This is obriously

$$
P\left(\Omega^{*}\right)=P\left(\bigcup_{u \in U} A_{u}\right)
$$

where $]_{u}=\left[\left(\omega_{l}, \omega_{k}\right): R(1)\left(u, \omega_{l}\right), \omega_{R}\right)=w_{2}$. If the $1_{u}$ 's are disjoint, as woukd be the case if no plant state $\left(\omega_{l}, \omega_{k}\right)$ is associated with more than one $"$, then this probability is simply

$$
P\left(\Omega^{*}\right)=I\left(\bigcup_{u \in l} \Lambda_{u}\right)=\sum_{u \in L} P\left(\cdot 1_{u}\right)
$$

Of course, before $P\left(\Omega^{*}\right)$ ean be detcrmined, a probability distribution must le associated with the sample space $\Omega_{l} \times \Omega_{k}$. This is accomplished by constructing the probability space $\left\langle\Omega_{D} \times \Omega_{R}, a_{I} \times a_{R}, P \Omega_{D}, \Omega_{R}\right\rangle$. This space must be consistemt with $\left.I\right)$ and $R$ in the sense that $\Omega^{*}$ must be a bona fide element of $a_{l} \times a_{R}$ so that its probability is well defined. This is why the measurability ( sec Section 4.1$)$ of $D$ ) and $R$, or at least of $D$, and $R$, is essential.

$\Lambda$ s another example, consider the probabilistic version of question 9 above: "What is the probability that at least one undetected sequence u causes diversion?" This probability is clearly

$$
P\left(\left(\Omega^{*}\right)_{d l=0}\right)=P\left(\bigcup_{u \in U} A_{u} \cap B_{u}\right)
$$

where $A_{1}$ is as before and

$$
\left.\left.B_{u} \stackrel{\text { DI: F }}{=}\left(\omega_{D}, \omega_{R}\right): I\right)\left(u, \omega_{D}\right)=(u, 0), u \text { fixed }\right]
$$

When probabilities of adversary acts are introduced, great care should be exercised not to develop a poor model of adversary behavior. Whereas the union of plant states usually makes sense when system failures are discussed, the union of adversary acts usually does not because an adversary cannot conmit several acts simultaneously. The question is thoroughly explained in Ref. 7 where additional probabilistic questions are formulated.

In conclusion to this chapter, it is evident that many other relevant questions can be precisely stated in this framework. In the context of safeguard system analyses, which rely heavily on combinations of sets of things (events, ...), we have attempted to illustrate the relevance of probability and set theory and their operations, and have provided a firm foundation for asking more complex questions. 


\section{THE STRUCTURED ASSESSMENT APPROACH (SAA) ANALYZFD IN OUR FRAMEWORK}

He structured ansessment approach $(S A N)^{8,9}$ is a methodology developed by lalwrence 1.nermore laboratory ower the past fell years os analyze nuclear safeguard systems. Informally. it is a multilenel hicrarchical structure. cach level taking a broader vicw of the salfeguard problem than the presious ane. At the lower levels, saleguard sy'stems arestudied in greater deterninistic and static detail Ai higher levels, probalijlistic and dynanic issues are addressed. In this chapter, in Sece tion 6.1, we address the deferministic ispects of $S A \Lambda$, which are discussed at Ievel I $\left(S A A_{1}\right)$. then, in Section 6.2, we address the probabilistic questions, treated at Ievel II $\left(S A A_{I I}\right)$, and we defer any discussion of higher lecels of analysis for future reperts.

\subsection{DHTERMINISTIC ANALYSIS: LEVEL I (SAA}

The purpose of level 1 analysis is to deterninc if a nontampering adversary who is assuncel not to know the plant state can divert nuclear materials without being detected by monitors assunced to work perfectly.

In this section, we define the outputs and structure of lecel I precisely. In the first few subsections we define the various basic ingredients of $S A A_{1}$, and in subsection 6.1 . ingredients into a formal definition of $S A \Lambda_{1}$

In the following discussion, let $l$, be the set of all facility elements with which an adiersary could potentially interact in some nanner, either through sineple encounter or through tampering (sce Ref. I for precisc definitions of such interactions). The set $l$ : could be portals, valves, monitors. tanks and their locations, arcas, matcrial acecss points, ctc. l.et $\Omega$ be the set of plant states (process conditions, flows, tank contents, operating modes, ctc.).

\subsubsection{Matcrial Access Points}

With cach adversary $t \in \mathscr{A}$, the set of all almissible adiersurics, is associated a set of material acess points for $d$, nap $1 \subset$ \&. Because these points may depend on the plant state, we model this association with the material aciess point functionl.

MAP :,$\quad \times \Omega \rightarrow 2^{l:}$, the set of all subscts of $t:$ The matcrial alcess points for $1 \in . \Omega$, and for a plant in statc $\omega \in \Omega$, arc

$$
M \wedge P(\lambda, \omega)=\operatorname{map}_{(\lambda, \omega)} \in 2^{-l}
$$

It is a sinple deduction that all admissible material access points associated with plant state $\omega$ are

$$
M(\omega)=\bigcup_{i \in A} M \Lambda P(A, \omega) \subset I:
$$

\subsubsection{Target Sequences}

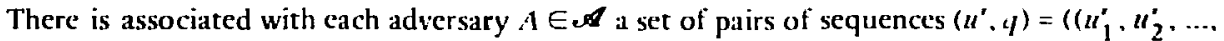
$\left.\left.u_{n}^{\prime}\right),\left(q_{1}, q_{2}, \ldots, q_{m}\right)\right)$ whose clements $u_{i}^{\prime}$ and $q_{j}$ are elements from $k$. A sequence $u$ represents a path of various elements in $E$ which an adversary encounters in secking nuclear materials. A sequence $q$ represents a path of material process clements or devices in $E$ whose state may be changed by an adversary following path $u$ '. Note that the meaning of "path" is very general here, and should be conceived as a graph-theoretic concept where it is a sequence of abstract nodes or vertices of a graph related by edges. Any node can, for instance, represent the location of an adversary or an act by an adversary, or even hotl. If the ordering in these sequences is immaterial, then these can be viewed as ordinary sets of crents, in which case wc express $\left(u^{\prime}, q\right)$ as 


$$
\left(u^{\prime}, q\right)=\left(u_{1}^{\prime}, u_{2}^{\prime}, \ldots, u_{n}^{\prime},\left\{q_{1}, \ldots, q_{m}\right\}\right)
$$

In Ref. 8, such pairs of sets are referred to as tanget sits. When ordering is retained, we shall refer to these as target seguences.

Because the number of material access points (map's) reletant to a particular adversary strategy is ustally small, such points may serve as "pivots" for an alversary's target sets, much like lig. 6-1. in this figure, $u_{i}^{\prime}$ nodes are adversary acts (actions at specific locations), and $q_{j}$ nodes represent the flow of special nuclear matcrials through the plant. One natural way to define target sequences, then, is to atssociate with each adversary, material-acesss-point pair $(A$, map) a path pair (sequence pitir) $\left(u^{\prime}, q\right)$. Realizing that this association tan depend on the plant state, we accomplish it with the tanget stequence selection function

$$
\mathrm{TS}: .11 \times \Omega \rightarrow E^{\prime \prime} \times E^{\prime \prime} \text {, }
$$

where $l^{\prime \prime}$ is the sct of all countable scyuences of clements in $l:$ including the empry seyuence. For any* $(1, m, \omega) \in ., \times . \| \times \Omega, T S(\Lambda, m, \omega)=\left(u^{\prime}, q\right) \in l^{\prime \prime} \times l^{\prime \prime}$, where $\|$ is the sec of all admissible

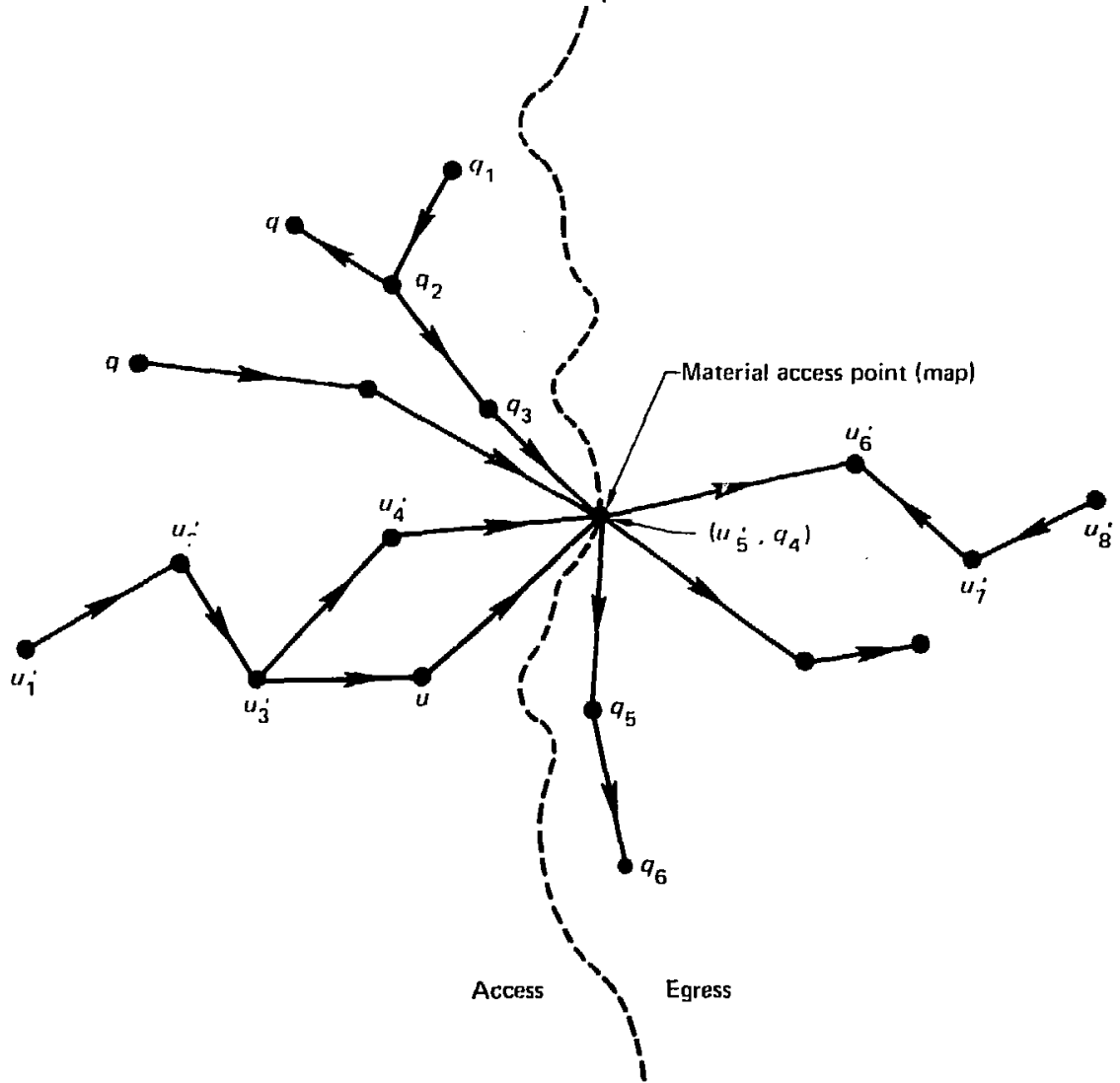

Fig. 6-1. The concept of a target sequence $\left(u^{\prime}, q\right)$, where $u_{i}^{\prime}$ nodes are adversary acts and $q_{j}$ nodes are flow of special nuelear materials.

* Unless this leads to confusion, we denote a material access point simply by $m$. 


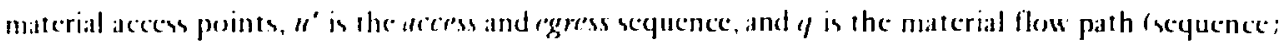
associated with $(A, m, \omega)$. Note that we allow only onc seywence pair $\left(u^{\prime}, 4\right)$ lour each adversary 1 This is a trival mathematical convenicnce. If another seyuence could te realized by it single "real" alversary, we simply introduce a "new" adversary, possibly artificial. If that is not salisfactory, we merely gencralize TS to a set-valued mapping, and all such objections disappear, at the expense of additional mathenatical conplexity. !urthermore, this tine point becones irrelevant when we discuss "generic" adversaries bater in this chapter.

It should be noted that wot every $(A, m, w)$ triple gives rise tes a nonempiy sequence because of the constraint that nest every adversary can aceess every point in $\|$. This constrant was expressed carlice in terms of the function $M \wedge P:, A \times \Omega \rightarrow 2$. We can incorporate this constraint into our target sequence selection map very simply by considering MAP as a relation $R_{\mathrm{MA}}$ on $\Omega \times \Omega \times 2^{\prime}$.

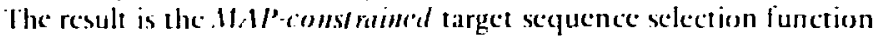

$$
T S_{M A P}: \mathscr{R}_{M A P} \rightarrow I .^{n} \times 1: n
$$

We then obrain the set of all larget seapuencess

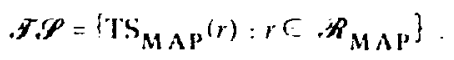

\subsubsection{Target Monitor Sets}

Some of the elements in l: are monicurs, devices whose principal purpose is to observe adversary acts directly or indirectly and generate certain signals when undesirable acts take place. $A$ basic idea in characterizing the purpose and performance of monitors is the field of vie' $w$, range, or coverage' of a monitor. (iiven that $l:$ is the universe of elenents or events of interest, this property of a monitor may be stated in terms of the set of elenents "covered by" or "protected by" the monitor. There are obviously many ways and degrees with which any object can be protected by another, and a complete description of this property may requirc sophisticated statist ical and mathematical analyses, particularly when several monitors act in concert to obscrec a certain set of things. When microwave monitors are considered, for instance, the distance of an object away from the monitor influences the degree to which the object is covered, and a trade off between loss of information and mudel cconomy must be made to decide whether microwave radiation patterns should be included in the model. We do not discuss such esoteric models. Rather, we take the binary approach where either a given element $e \in l$ is within the field of view of a monitor $m \in l$, or it is not. We include the state space $\Omega$ in our characterization because some monitors may be inoperative for some plant states $\omega$ (maintenance mode, for instance). The result is simply a ficlit-of-vicw function*

$$
\text { MON : Mon } \times \Omega \rightarrow 2^{l:} \text {, }
$$

where Mon $\subset E$ is the set of monitors. For each monitor mon $\in$ Mon and state $\omega, \operatorname{MON}(\operatorname{mon}, \omega)$ is the set of elements within the field of view of ("covered" by) mon. We shall occasionally refer to this set as $E_{\text {mon }}$. viously

The set of monitors that cover or protect a given element $c \in E$ for appropriate states $\omega$ is ob-

$$
\operatorname{Mon}_{e}=\operatorname{MON}^{-1}(\{e\}) \subset \operatorname{Mon} \times \Omega
$$

- Matrices may be used descriptively, but they are not as convenient as funcrional representations, with which inverses and other standard operations are handled in a simpler manner. 
The moniturs that reach a given element $c \in t:$ when the state is $w$ are

$$
\left.\left.\operatorname{MON}^{1}\left({ }^{2}\right\}\right)_{\omega}=\text { imon } \in \operatorname{MON}:(\text { mon, } \omega) \in \operatorname{MON}^{-1}(\{\}), \omega \text { fixed }\right) .
$$

For any target sequence $\left(\left(u_{j}^{j}, u_{2}^{\prime}, \ldots, u_{n}^{\prime}\right),\left(q_{1}, \ldots, q_{m}\right)\right)$, the set of monitors that reach at least one e'ement $u_{j}^{\prime}$ ot $q_{j}$ when the state is $\omega$ is

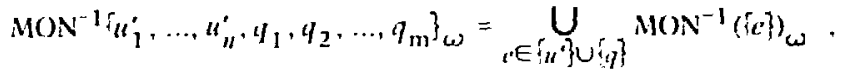

where

$$
\left\{u^{\prime}\right\}=\bigcup_{i=1}^{\prime \prime}\left\{u_{i}^{\prime}\right\},\{q\}=\bigcup_{i=1}^{m}\{q,\} .
$$

If $\operatorname{TS}(A, m)=\left(a^{\prime}, q\right)$, we definc

$$
[\mathrm{IS}(A, m)] \text { by }\left\{u^{\prime}\right\} \cup\{q\}
$$

So MON ${ }^{-1}\left(\left\{u^{\prime}, q\right\}\right)_{\omega}$ is the target monitor set (TMS) for target set $\left\{u^{\prime}, q\right]$ when the state is $\omega$.

$\Lambda$ principal output of a "Level I" analysis in the structured assessment approach $\left(S A A_{1}\right)$ is a listing of target monitor sets, empty or not, for all adversary types. Clearly, any iime an empty set is produced, the target set in question is not covered, and such uncovered sets are especially of interest and listed as well.

To show how various generic types of adversary show up in such an output, we bring to bear the various mathematical constructs introduced above.

Definition. The target monitor set (TMS) for adversary $A$, access point $m$, and state $\omega$ is the set

$$
\operatorname{TMS}(A, m, \omega)=\underset{\iota \in[\operatorname{TS}(A, m, \omega)\}}{\bigcup} \operatorname{MON}^{-1}\left(\{c)_{\omega}\right.
$$

This set can be verbally described by using the existential interpretation of the union operator $U$. Any monitor mon is a member of the target monitor set for $A, m$, and $\omega$ if and only if (iff) there exists an clement $e$ in the target set $t=\operatorname{TS}(A, m, \omega)$ which is in the field of view of the monitor mon.

Since, for a fixed plant state $\omega$, each adversary $A$ has a set of material access points $\operatorname{MAP}(A, \omega)$ $\subset E$, the target monitor set (TMS) for $A$ and plant state $\omega$, regardless of which $m$ the adversary chooses, is

$$
\operatorname{TMS}(A, \omega)=\bigcap_{m \in M \operatorname{AP}(A, \omega)} \underset{e \in\{\operatorname{TS}(A, m, \omega)\}}{\bigcup} \operatorname{MON}^{-1}(\{e\})
$$

Verbally, any monitor mon is a member of $\operatorname{TMS}(A, \omega)$ iff, for all material access points $m$ for $A$, there exists a fixed element $e$ in $\operatorname{TS}(A, m, \omega)$ which is in the field of view of mon. Clearly, if each adversaiy has only one material access point, the intersection operation is superfluous. It is important to comment here that the definitions of TMS in Refs. 8 and 9 are not clear. If a given adversary has more than one $m$, then merely taking the union of all monitors covering at least one element of 
each target eet does not indicate whether the adversary is covered or not: cerry material access point secpuence $1 \wedge(A, m, \omega)$ must be covered individually. $\wedge$ geod way to characterize this is by listing the TMS's for each adversary and cach of his matcrial access points, thus

$$
\operatorname{TMS}(A, \omega)-\because \operatorname{MS}(A, m, \omega): m \in M A P(A, \omega) !
$$

where

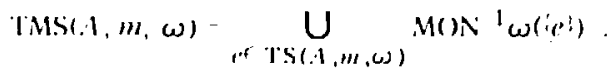

Adversary 1 is then colered (when the system is in state $\omega$ ) just in cisc nese of the sets TMS(A, $m, \omega)$ are enpty (for fixed $\omega \in \Omega$ ). This is the set we shall ident ify as the target monitor set of adversary $A$ when the system is in state $\omega$.

$A$ generic adversary is a diss $f$ ol tementary alversarics $d \in, \mathscr{A}$. For such an adversary, we have the following definition:

Definition. The target monitor set (TMS) for generic adversary $c_{\text {i }}$ when the state is $\omega$ is the set

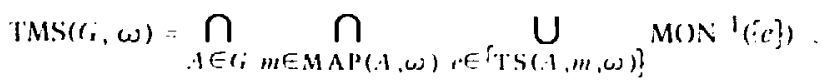

'S.re we have the added constraint that, regardless of which specific form $A \in$; the generic adversary $C ;$ takes, and regardless of which material aceess point $m$ he uses, any monitor in TMS( $;, \omega)$ must "cover it." Any monitor mon $\in$ Mon is thus a member of TMS( $;, \omega)$ itf for all specilie instanriations $A$ of $(;$, and for all material acess points $m$ for 1 , there exists a fixed element,$\in\}(A, m, \omega)$ ) which is in the field of view of mon. This element is "fixed" in the same sense as $\omega$. it does not change with $A$ or $m$.)

Finally, we are interested in the set of target sequences that are unmonitored or not covered. Recall that the target sequence for adversiry $A$ and one of his material access points $m$ is "TS(A, $m, \omega)$ $=t \in l^{\prime \prime} \times l^{n}$. For a given plant state $\omega$, al target sequence $/$ is mot coverd by . Hon iff MON $\left.{ }^{-1}(? t\}\right)$ $=\bigcup_{l \in\{t\}} \operatorname{MON}_{\omega}^{-1}(\{c\})=0$.

\subsubsection{Monitor Null Sets}

We define the monitor null set of an adversary $A$ when the plant state is $\omega$ as

$$
\operatorname{Null}(A, \omega)=\left\{t=\operatorname{TS}(A, m, \omega): \operatorname{MON}_{\omega}^{-1}(\{t\})=\emptyset, m \in \operatorname{MAP}(A, \omega)\right\}
$$

These are the sequences associated with the access points $m$ of adversary $A$ that are not covered by Mon when the facility state is $\omega$.

For a generic adversary $(i \subset . A$, the monitor null set is

$$
\operatorname{Null}(G, \omega)=\bigcup_{A \in G}\left\{t=\operatorname{TS}(A, m, \omega): \operatorname{MON}_{\omega}^{-1}(\{t))=\emptyset, m \in \operatorname{MAP}(4, \omega)\right\}
$$




\subsubsection{Sunmary of $S A A_{1}$ in Our Framework,} and Sonc Qualitative Performanec Measures

Now that we have discussed and furmalized all the basic ingredients of $S A A_{l}$, we can provide a formal set-theoretic definition of $S A A_{1}$ as an octuple:

$$
S \Lambda \Lambda_{1}=\langle, A, I S \Omega, M, M A P, T S, M O N, M O N\rangle
$$

where
.A is a class of adversarics (a gemeric adversiary is a subset $(; \subset . A$ ).
$E$ is a set of ilements (components, ...).
$\Omega$ is a set of system states,
$\| \subset \subset$ is a set of matcrial access points (map),
MAP : $A \times \Omega \rightarrow .11$ is the material access point (map) specification finction,
TS : $A \times M \times \Omega \rightarrow l: "$ X $\times 1: "$ is the target set specification function,
Mon $C E$ is the set of monitors,
MON : Mon $\times \Omega \rightarrow 2^{l:}$ is the fichlof-vicu specification function.

We can now define two basic performance measures used in the $\mathrm{S} \Lambda \Lambda_{1}$ for a safeguard system $S C ; \in S$ in a particular statc $\omega \in \Omega$. The first measure is a function

$$
\prod_{N \cup L L(\omega)}: S \rightarrow 2^{1: "} \times 1: ",
$$

which associates with each safeguard system $S G \in S$ the monitor null set for all adversaries in $\not \Rightarrow$ when $S C ;$ is in state $\omega$. This sel is

$$
\operatorname{Null}(\omega)=\bigcup_{l \in, \not d} \operatorname{Null}(A, \omega)=\bigcup_{d \in \mathscr{R}}\left\{t \operatorname{TS}(A, m, \omega): \operatorname{MON}_{\omega}^{-1}([t\})=\emptyset, m \in \operatorname{MAP}(A, \omega)\right\}
$$

Thus, for every $S G$ in $S$, 'NULL( $\omega)(S G)=N u l l(\omega)$, the collection of target sequences $t$ that are not covered by any of the monitors in Mon when the system is in state $\omega$.

The second performance measure is an aggregated measure which represents the situation where the existence of even a single uncovered $\mathrm{T}$ is used as a criterion for rejecting a safeguard system SG. This measure is an aggregation of ${ }^{N}{ }_{N L L}(\omega)$ and is itself a function $\Phi_{N U L L(\omega)}^{\prime}: S \rightarrow\{0,1\}$ defined l)y

$$
\Phi_{\mathrm{N} \cup L L(\omega)}^{*}(\mathrm{SG})=\left\{\begin{array}{l}
0 \text { if } \Phi_{\mathrm{N} \cup L L(\omega)}(\mathrm{SG}) \neq \emptyset \\
1 \text { otherwise }
\end{array}\right.
$$

We have discussed a safeguard system in a particular state $\omega \in \Omega$. It should be evident that we could readily analyze more general questions, as we did in the first chapter. By using set operations, for instance, we could model such statements as "for all states," "regardless of the system state $\omega$," "for at least one state," "the minimum over all states," and so on. $\Lambda$ t this point of SAA development, however, such aggregated results are not produced, and monit.* null sets are generated for each of a set of operating states ("modes") of interest. 


\subsection{PROHA HILISTIC ANAIYSIS: LEVEL II (SAA}

"Level II" analysis is an extension of level 1 analysis in the sense that randon failures of the monitors are considered, and the adversary may be assumed to know the plant state.

\subsubsection{Level II Analysis: The $S A \Lambda_{\text {II }}$ System}

Recall from our detcrministic analysis of SA (Section 6.1) that the set of all monitors cosvering is target sequence is

$$
\operatorname{TS}(A, m, \omega)=\bigcup_{, \in \in \operatorname{TS}(A, m, \omega)} \operatorname{MON}_{\omega}^{-1}(\dot{q}(\omega)
$$

where

$A$ is some adversitry,

$\omega$ is a detection sulssystem state ,

$m$ is a material access point for $A$.

In this chaper, we extend the analysis to determine the pre' ability of detcetion of various adversaries $A$ in our franework in terms of the infornal concepts developed in $S A A$. To this end, we modify our detection sulsystem $D: U^{\prime} \times \Omega \rightarrow V^{\prime}$ slightly (no tampering) by attaching to $\Omega$ a probability structure ( $(a, P)$, where $a$ is a collection of subsets of $\Omega$ (the cents) and $l$ is a probability distrilumtion or measure on $Q$. The sample space $\Omega$ is now part of the experiment or probability space $\langle\Omega, a, l\rangle$. Using this probabilistic lramework, we find the probability that every target sequence $T S(\lambda, m, \omega)$ is covered is

$$
P\left\{\omega: \bigcup_{c \in \operatorname{TS}(A, m, \omega)} M() N_{\omega}^{-1}(\{, j) \neq \omega\} .\right.
$$

The probability $P_{D, A}$ that a given adversary $A$ is detected or "covered" is the probability that he be detected regarlless of which material aceess point $(m)$ he chooses, or the probability that all his paths be covered. Realizing that the selection of a material access point by adversiry $A$ is itself a random variable $M \Lambda P_{,}: \Omega \rightarrow H$, we must use, for each $m \in N$, the joint enent that $m$ be a material access point for $A$ and that the associated target set $\operatorname{TS}(A, m, \omega)$ be covered. This event, which is an clement in $Q$, is

$$
E_{m}(A)=\{\omega: m \in \mathrm{MAP}(\Lambda, \omega)\} \cap\left\{\omega: \underset{e \in \operatorname{TS}(A, m, \omega)}{\bigcup} \operatorname{MON}_{\omega}^{-1}(\{c\}) \neq \emptyset\right]
$$

We finally obtain the complete expression for the probability of detection of $A$ :

$$
P_{D}(A)=P\left(\bigcap_{m \in M} E_{m}(A)\right)
$$

If $A$ has only one access point $m_{A}$, then a much simpler expression obtains:

$$
\left.P_{D, A}=P\left\{\omega: \underset{\epsilon^{\prime} \in \operatorname{Ts}\left(A, m_{A}, \omega\right)}{\bigcup} \operatorname{MON}_{\omega}^{-1}(\{e]) \neq \not\right]\right\}
$$

It is unfortunate that, even if the complementary measure $1-P_{D, 4}$ (the probability of no detection) is considered, no convenient decomposition of $P_{D, A}$ appears to exist as in the case of 
SVAM. This is in part dese to the intuitive but heuristic manner in which the various clements of SA $A$ were developed. In particular, lecause monitors can "see" several elements in f, and adversaries can have scicral material access points, the result is ste-vilmed maps MON and MAP. The resulting intersections berween adversaries, monitors, and other facility elements considerably complicate the tast of decomposing detection or diversion cents into suherents whose probabilitics might be casier to assess. This lack of structure may become a computational drawback when large realistic facilities are considered. If we are interested in merely listing the probabilities of detection for a set of monitor sets ${ }^{8,9}$ and for a given adversary-this could be ciewed as a reliability index for the system-then if the selection of aceess points is independent of the operation of the monitors, we would obtain the collection of probabilitics

$$
\left.H_{m}: m \in H^{\prime} \subset-11\right\}
$$

where

$$
\left.\left.P^{\prime \prime \prime}=P^{\prime} \omega: \bigcup_{, \in T S(1, m, \omega)} \operatorname{MoN}_{\omega}^{1}\left(c_{c}\right\}\right) \neq 0\right]
$$

and $I^{\prime}$ is some collection of material access points.

If a generic adversary $C_{i}$ is of interest, the probability of derecting such an adversary is simply

$$
r_{H, r}=r\left(\bigcap_{i \in r_{i}} \bigcap_{m \in \| U} I_{m}(\cdot)\right)
$$

where $l_{m}$ is defined above, and each $A$ is an element of the generic type ("cliss") $(i$.

\subsubsection{Adversaries Who Exploit Their Knowledge}

of the Plant Operating Mode

In choosing between alternative action strategies $u \in U$, the adversary will obviously be influenced by any privileged information he may possess about the safeguard system and the plant in which it operates. One of the plant "states" about which the adversary may possess such information is the plant operating mode $\omega_{O M}$, a subcollection of components of $\omega$ (day or night operation, audit, fill mode, ...). For purposes of analysis, a good way to represent this information in the decision process is to assume that the adversary will choose different actions for different plant modes. If this were not the case, "plant mode" might not be such an important parameter after all, or the various modes might have been too finely subdivided. The definition of these modes thus depends to some extent on the class of adversarics considered.

We then assume that the plant mode parameter $\omega_{O M}$ partitions the action space $U$ into $k$ equivalence classes $\left\{U_{k}: k \in K\right\}$, one $U_{k}$ for cach plant mode $\omega_{\mathrm{OM}}=\omega_{k}$. This partition could be obtained by analyzing the action-state diversion graph of Fig. 5-1, Chapter 5 . If the system state vector is $\omega=\left(\omega_{\mathrm{OM}}, \omega_{c}\right)$, where $\omega_{c}$ is the component state variable, we can now remove the $\omega_{\mathrm{OM}}$ parameter as a random event or condition, and state that, if the adversary chooses $u \in U_{k}$, then the plant or safeguard system is in operating mode $\omega_{O M}=\omega_{k}$ for sure, and conversely. The only remaining uncertainty is now associated with the component state variable $\omega_{c}$. Note that the knowledge of $\omega_{\mathrm{OM}}$ only enbances the chances of success of the adversary, and does not necessarily guarantee his success. In terms of the SAA, this special property is characterized as a constraint on the target sequence selection map TS : $A \times M \times \Omega \rightarrow E^{n}$. We break the state space $\Omega$ into two parts, $\Omega_{c}$ and $\Omega_{O M}$, where $\Omega_{c}$ are the component states and $\Omega_{O M}$ are the operating modes. 
When the adversary knows nothing about the operating mode of the plant, then the target selection map TS : $\Omega \times M \times \Omega_{r} \times \Omega_{O M} \rightarrow l: "$ would be highly insensitice to $\omega_{O M} \in \Omega_{O M}$. If it is complerely insensitive to it, of course, we obtain

$$
\operatorname{TS}\left(A, m, \omega_{c^{\prime}}, \omega_{O M_{1}}\right)=\operatorname{TS}\left(A, m, \omega_{c}, \omega_{O M_{2}}\right)
$$

for all $A, m$ and for all $\omega_{O M_{1}}$ and $\omega_{O M_{2}}$ in $\Omega_{O M}$.

When the adversary knows the plant operating mode and can exploit that knowledge, then the opposite situation tends to arise: with cach $\omega_{O M} \in \Omega_{O M}$ is associated a "preferred" subset of $/:^{\prime \prime}$. In other words, the $\omega_{\mathrm{OM}}$ variable will shift or skew the distribution of TS to an extent which depends on the probability distribution on $\Omega_{c}$ and the sensitivity of TS to $\omega_{c}$. In the case where rS is insensitive to $\omega_{c}$, or $\omega_{c}$ is not randon, TS is deterministic and values of $\omega_{O M}$ fully specity sequences of acts in $l^{\prime \prime}$ for a given $A$ and $m$. It should be clear that, in any casc, the uncertainty associated with $\omega_{O M}$ (the distribution $/ \Omega_{O M}$ ) becones irrelevant in describing this type of adversary, and the uncertainty associated with detection may be dramatically reduced. 


\section{THE SYSTEMS VULNERABILITY ASSESSMENT METHOD (SVAM) ANALYZED IN OUR FRAMEWORK}

rr the past few years, a digraph fault-tree methodology has been developed at Lawrence Livers. re Laboratory to analyze nuclear safeguard systems. This method, recently named SVAM (system, vulnerability assessment method), is a deterninistic tool to compute the adversary action and systenl state triples $(a, 0, \omega)$ that lead to theft or diversion of special nuclear materials (SNM). Unfortunately, not nuch has been written to document or report this method in the safeguards and systems analysis literature, and only one bricf reference ${ }^{10}$ was available describing its purpose and outputs. Any discussion of this method here is the result of our interpretation of Ref. If and our private discussions with some of its authors.

\subsection{DETERMINISTIC ANALYSIS}

In rhis section, we describe the outputs of SVAM and express these in terms of our framework and some of the questions we posed in Chapter 5. Here we show that these outputs imply the use of certain qualitative performance measures, which we describe.

SVAM proluces four categories of "event sets" which describe all possible ways for a particular adversary to perpetrate SNM theft; the definitions of these categories we reproduce here verbatim from Ref. 10:

$S_{1}=$ those cvent sets of SNM theft with no detection by the safeguards system, only random failures occurring

$S_{2}=$ those event sets of SNM theft with inidequate safeguards system response, only random failures occurring

$S_{3}=$ those event sets of SNM theft with no detection by the safeguards system, adversary tampering occurring

$S_{4}=$ those event sets of SNM theft with inadequate safeguards system response, adversary tampering vocurring.

It is not clear what is neant by "those event sets" in all the above categories. For instance, the definitions of $S_{2}$ and $S_{4}$ do not state whether all cvents are detected, or whether detection is relevant at all. In addition, the statement concerning inadequate response is redundant since an SNM theft implies it. In $S_{3}$ and $S_{+}$, it is not clear whether "random" failures are also occurring or whether the plant or facility is in a given operating or failure state during the tampering and other diversion acts.

$\Lambda$ s a result of recent discussions, ${ }^{11}$ we shall assume that the sets $S_{1}$ and $S_{2}$ are collections of "basic"* adversary acts (events) and failures (events), and we include these as possible components of our adversary sequences $u$ and states $\omega$. We shall generally make reasonable assumptions about the intended meaning of $S_{1}$ and $S_{2}$ to avoid the inconsistencics mentioned above (similarly for $S_{3}$ and $S_{4}$ ). Note that the inclusion of nontampering adversary acts in $S_{1}$ and $S_{2}$ is not mandatory, and we may simply specialize $S_{1}$ and $S_{2}$, or even interpret $S_{1}$ and $S_{2}$, as sets of plant and component states for which diversion is possible for some adversary strategy. This would be analogous to confining our discussion to the sets $\left(\Omega^{*}\right)_{d=0}$ and $\left(\Omega^{*}\right)_{d=1}$ rigorously developed in Chapter 5 .

\subsubsection{The Collection $S_{1}$ of Events}

We interpret $S_{1}$ as a set of triples $\left(u, \omega_{D}, \omega_{R}\right)$, where

$u=\left\{u_{1}, \ldots, u_{n}\right\}$, a set of inputs, the adversary actions,

$\omega_{D}=\left\{\omega_{D 1}, \ldots, \omega_{D n}\right\}$, a set of detection subsystem states,

$\omega_{R}=\left\{\omega_{R 1}, \ldots, \omega_{R t}\right\}$, a set of response subsystem states,

\footnotetext{
Elements of minimal cut sets of fault trees, for example.
} 
and $u$ and $t$ are natural numbers. In this setting, each $\left(u, \omega_{D}, \omega_{R}\right)$ in $S_{1}$ represents three sets of things that must occur for diversion to take place under conditions of no detection of any $\left(u, \omega_{l}\right)$ pair. Thus, for each $\left(u, \omega_{D}, \omega_{R}\right) \in S_{1}$, the first two sets $\left(u, \omega_{D}\right)$ must be such that $D\left(u, \omega_{\nu}\right)$ $=(u, 0)$ (no detection). As we mentioned in our introduction, each of these sets is merely an unordered collection of events, inputs, and states. The order in which events occur is thus not discussed with SVAM, at least in its present form. With the appropriate interpretation, therefore, our set (Div) $d=0$ of Chapter 5 corresponds to $S_{1}$.

\subsubsection{The Collection $S_{2}$ of Events}

Since no mention is made of detection constraints, it should be assumed that $S_{2}$ contains both the detected and undetected event sets that lead to diversion. This causes an intersection between $S_{1}$ and $S_{2}$ which complicates the decomposition unnecessarily; however, we shall assume that only detected sequences are contained in $S_{2}$. The corresponding set in our framework is thus simply (Div) $d=1$.

\subsubsection{The Collection $S_{3}$ of Fvents}

Although failure or plant states are not mentioned in defining $S_{3}$ (Ref. 10), we assume that the occurrence of tampering does not preclude random failures from occurring, and we include the plant state $\left(\omega_{1}, \omega_{R}\right)$ in our version of $S_{3}$. and

We interpret $S_{3}$ as a set of 5 -tuples $\left(u, \omega_{D}, 0_{D}, \omega_{R}, 0_{R}\right)$, where $u, \omega_{D}$, and $\omega_{R}$ are as before,

$$
\begin{aligned}
& \theta_{D}=\left[\theta_{D 1}, \ldots, \theta_{D q}\right] \text {, a set of structural parameter values describing the relevant } D \text { - } \\
& 0_{R}=\left[0_{R 1}, \ldots, 0_{R s}\right] \text {, a set of structural purameter values describing the relevant } R \text { - }
\end{aligned}
$$

The no-detection constraint now implies that for each $\left(u, \omega_{D}, \theta_{D}, \omega_{R}, \theta_{R}\right) \in S_{3}$, the first three sets $\left(u, \omega_{D}, 0_{D}\right)$ must have the property that $D\left(u, \omega_{D}, 0_{D}\right)=(u, 0)$ (no detection). Thus, $S_{3}$ corresponds to our set (Div) TAMP $(D, K), d=0$ of Chapter 5 .

\subsubsection{The Collection $S_{4}$ of Events}

Again no mention of detection is made in defining $S_{4}$ (Ref. 10), but we shall assume that detection is intended. The corresponding set in our framework is thus simply $(D i v)_{T, A M P}(D, R), d=1$ of Chapter 5.

\subsubsection{Summary of SVAM Outputs, and Some \\ Deterministic Peformance Measures}

To summarize, the outputs of SVAM, as interpreted from Ref. 10, imply the use of the following performance measures ( $S$ is a collection of safeguard systems of interest with elements $S G$, as discussed in Chapter 2:

1. The first measure $\Phi_{1}$ extracts from any safeguard system $S G \in S$ the collection of event sets $S_{1}$. Thus,

$$
\Phi_{1}: S \rightarrow 2^{U \times \Omega_{D} \times \Omega_{R}}
$$

is defined by

$$
\Phi_{1}(S G)=(\text { Div })_{d=0}=S_{1} \text { for system } S G \in S .
$$


2. The second measure $\}_{2}$ cxtracts from any $S\left(; \in S\right.$ the coliection of events $S_{2}$. Thus,

$$
\mathbb{1}_{2}: S \rightarrow 2^{U \times \Omega_{D} \times \Omega} R
$$

is defined by

$$
\Phi_{2}(\mathrm{SG})=(\mathrm{Div})_{d=1}=S_{2}, \mathrm{SG} \in S \text {. }
$$

3. The third measure $\Phi_{3}$ extracts $S_{3}$. Here

$$
\Psi_{3}: S \rightarrow 2^{U \times \Omega_{D} \times \Theta_{D} \times \Omega_{R} \times \Theta_{K}}
$$

is defined by

$$
\Phi_{3}(S G)=(\text { Div })_{T} \wedge M P(D, K), d=0, \text { for any } S G \in S \text {. }
$$

4. Finally, the fourth measure $W_{4}$ extracts $S_{4}$. Herc

$$
\Psi_{4}: S \rightarrow 2^{U \times \Omega} D \times \theta_{D} \times \Omega_{R} \times \Theta_{R}
$$

is defined by

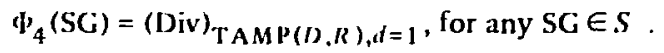

\subsection{PROBABILISTIC EXTENSIONS TO SVAM}

In this section, we develop an expression for the probability of diversion for the various detection and response subsystem conditions associated with the sets $S_{1}, S_{2}, S_{3}, S_{4}$ produced by SVAM. The expressions we obtain are simple additive forms which are exact: no assumptions of independence and repeatability of the events ${ }^{10}$ or any other approximations are needed for their validity. Hence, they differ substantially from the performance measures reported in Ref. 10 , which we show to be inappropriate. One of the safeguard system performance measures we obtain is also simply the sum of the performance measures of the subsystems $D$ and $R$. In this chapter, we assume that adversary and plant operating descriptions are complete: we do not sllow "don't care" conditions, and every component of the action or state sequence (vector) inust be specified exactly. For large but realistic systems, such complete descriptions obviously lead to combinatorial problems beyond the reach of any modern computer. Because of this limitation, and because many scenarios are described in terms of don't-care events or components, we have developed a new technique called STOP (superfast technique for obtaining probabilities). This method allows the computation of reliabilities and probabilitics of detection or diversion to any degree of accuracy, and for arbitrarily dependent and intersecting (not mutually exclusive) scenarios having arbitrarily many don't zare conditions. This new technique is reported in Ref. 7.

With the clearly stated assumptions of Chapter 7 , the four sets $S_{1}, S_{2}, S_{3}, S_{4}$ were shown to be equivalent to our four sets (Div) $d=0,\left(\right.$ Div) $d=1$, (Div) $\operatorname{TAMP}(D, R), d=0_{1}$, and (Div) T AMP $(D, R), d=1_{1}$, respectively.

It is important to recall from Chapter 3 that our analysis and our developments of performance measures are independent of whether or not adversary actions $u$ and tampering acts $\theta$ are explicitly included. We can simply let $U$ and $\Theta$ be empty sets, and concentrate on the plant or component 
state space $\Omega=\Omega_{l} \times \Omega_{R}$. We would then merely investigate probabilities associated with the sets $\left(\Omega^{*}\right)_{d=0}$ and $\left(\Omega^{*}\right)_{d=1}$ defined in Chapter 7 .

\subsubsection{Probability of Diversion and System}

Reliability as Safeguard Performance Measures

The principal probabilistic issuc in SVAM consists of computing the probability of these four sets, thereby olstaining a probability of diversion or system unreliability under the four detection conditions* described in Section 7.1. Since these probabilities are determined by the probability of uccurrence of individual puints in our corresponding sets, we define thesc points as events in the assuciated experiments. Considering the input experiment or space $(U, \mathscr{U}, P)$, for instance, if $u \in U$, then $\{u\} \in \mathcal{M}$. Before we compute these probabilities, we recall some elementary facts. For two events $A$ and $B, A \cup B=\mathscr{C}(\mathcal{P}(A) \cap \mathscr{C}(B))$ (De Morgan), and $P(A \cup B)=P(A)+P(B)-P(A \cap B)$. If $A$ and $B$ are independent, $P(A \cup B)=1-P(\mathscr{E}(A)) P(\mathscr{B}(B))=1-(1-P(A))(1-P(B))$. For a countable sequence of independent events $\left(A_{1}, A_{2}, \ldots\right)$,

$$
P\left(\bigcup_{i=1}^{\infty} A_{i}\right)=1-\prod_{i=1}^{\infty}\left(1-P\left(A_{i}\right)\right)
$$

For each of the four sets produced by SVAM, the 3-tuples $\left(u, \omega_{D}, \omega_{R}\right)$ or 5-tuples $\left(u, \omega_{D}\right.$. $0_{D}, \omega_{R}, 0_{R}$ ) are mutually exclusive: the adversary cannot complete two or more scenarios simultaneously, even if he makes multiple attempts. If collusion is involved, we resort to vectorial processes $\vec{u}=\left(\vec{u}_{1}, \ldots, \vec{u}_{n}\right)$ and $\vec{\theta}=\left(\vec{\theta}_{1}, \ldots, \overrightarrow{0}_{n}\right)$. This mutual exclusivity also holds in analyses which do not explicitly account for adversary acts of any kind, and merely encompass the system states (operating or failure conditions, for instance) in which diversion could occur. The system cannot be in two states at once! It is important to note that this cxclusivity condition liolus not only in our framework where adversary acts and plant states are n-tuples, but in other frameworks such as SVAM and SAA where the ordering is immaterial and adversary acts and plant conditions are completcly described by sets of events. The basic issuc is that the purpose of these "event sets" is to capture joint conditions which must hold for diversion to take place. More specifically, each such sct describes a specific "way" for a particular type of adversary to perpetrate SNM theft. 10,11 . . important point must be emphasized here. The mutual exclusivity condition is based on the assumption that the states of the system and the diversion event scts (states for which diversion occurs) are complete descriptions. Each of these descriptions must include a specific statement or event about every component of the system, whether the component is operative or not. Thus, the statement "diversion occurs when the first two component states are $\omega_{1}=a$ and $\omega_{2}=b$, regardless of $\omega_{3}$, $\omega_{4}, \ldots, \omega_{n}$," is not allowed. Every state description must be a specific $n$-tuple or a set of $n$ events for this introductory approach. In another report, ${ }^{7}$ the problem of conputing the probability of an arbitrary union of events is analyzed in detail.

We assume that the state of a system is determined by the state of its components, and we model each component as a binary random variable on a common sample space $\Omega$. Hence, each component is a random variable $E_{i}: \Omega \rightarrow[0,1]$, and may be viewed as a random event which occurs (the component operates properly) if $E_{i}=1$, and does not occur (the component fails) if $E_{i}=0$. We call the value of $E_{i}$ the state of component $i$ and define the state of the system as the $n$-tuple $\left(a_{1}, a_{2}, \ldots, a_{n}\right)$, where $a_{i}$ is the state of component $i$. Unless there is a possibility of confusion, we shall view $E_{i}$ as an event or a random variable, whichever is appropriate.

Now, the event sets in SVAM consist of basic cvents $E_{i}$ with which probabilities (the $q_{n}$ 's in Ref. 10) are associated. These events are thus also formally members of some underlying algebra of events, implicitly or explicitly, and are called basic because they are the primitive and indivisible

"Due to the complexity and size of these event sets, SVAM does not include a method for computing these probabilities at this time. 
components which constitute the sets $S_{1}, S_{2}, S_{3}, S_{4}$ in SVAM. Given such a collection $\left\{E_{i}: i\right.$ $=1,2, \ldots, n]$ of $n$ basic events which completely determine a diversion "way," it is the joint occurrence of these events that causes diversion, hence it is the joint event ${ }^{*} E_{1} \wedge E_{2} \wedge \ldots \wedge E_{u}$ that is of intcrest to us.

If we view all the cvents as sets, we obtain the joint event

$$
\because=\bigcap_{i=1}^{n} E_{i}
$$

the intersection of the $E_{i}$ 's. Now, if several $(m)$ such joint events $\left\{F_{1}, \ldots, F_{m}\right\}$ lead to diversion, and we are interested in the probability that diversion occurs regardless of which $F_{j}$ 's give rise to it, it suffices to determine the probability of the event $F_{1} \vee F_{2} \vee \ldots \vee F_{m}$. Expressed in terms of set theory, this is the event

$$
F=\bigcup_{i=1}^{m} r_{j}
$$

So we wish to find the probability

$$
r\left(\bigcup_{j=1}^{m} F_{j}\right), \text { where } F_{j}=\bigcap_{i=1}^{n} E_{i j} .
$$

The proposition below shows that the $F_{j}$ 's are all disjoint, and

$$
P\left(\bigcup_{j=1}^{m} F_{j}\right) \text { is simply } \sum_{j=1}^{m} P\left(F_{j}\right) \text {. }
$$

Although we consider only those cvents which completely describe the state of operation of a system and its components, the extension to the case where adversary acts are specifically included is simple. But note that, while unions are meaningful in a reliability analysis, great care must be exercised in using unions when adversary acts are included. When the probability of diversion is computed, taking the probability of the union over a collection of adversary acts doesn't make sense if the adversary cannot complete multiple scenarios simultaneously. This point is studied in greater detail in Ref. 7.

Proposition. Consider an experiment $\mathscr{E}=\left\langle\Omega, a, P_{\Omega}\right\rangle$ and a system with $n$ components $S=\left\{E_{i}: i=1,2, \ldots, n\right\}$, where each component is a binary random variable $E_{i}: \Omega \rightarrow\{0,1\}$ whose values are the state of component $i$. Assume all the subsets of $\Omega$ are events, thus the event algebra is $a=2^{\Omega}$ (all subsets of $\Omega$ ), and $P$ is some probability measure on $a$. Define the state $a$ of the system as the $n$-tuple $\left(a_{1}, a_{2}, \ldots, a_{n}\right)$, where each $a_{i}$ is the state of component $i, i=1,2, \ldots, n$ (Ref. 12 , pp. 2, 269). Thus, for each outcome $\omega \in \Omega$ of the experiment $\mathscr{E}$, i.e., for each "state of nature" $\omega$, the system $S$ is in state $a=\left(E_{1}(\omega), \ldots, E_{n}(\omega)\right)$. Let $P(a)$ be the probability that the system is in state $a$.

Then any two distinct system states $a$ and $b$ are mutually exclusive, and $\operatorname{Prob}(a \vee b)$, the probability that the system is in either state $a$ or $b$, is $P(a)+P(b)$.

\footnotetext{
$\because \Lambda, V$, and 7 are the conjunction, disjunction, and negation operators in standard propositional logic, whereas $n$,
} $\cup$, and $\mathscr{C}$ are the corresponding intersection, union, and complementation operators of set theory. 
Proof. I.et

$$
\begin{aligned}
a & =\left(a_{1}, \ldots, a_{n}\right), b=\left(b_{1}, \ldots, b_{n}\right), \\
P(a) & =I_{\Omega}\left\{\omega: I I_{1}(\omega)=a_{1}, \ldots, I:_{n}(\omega)=a_{n}\right\} \\
& =I_{\Omega}\left(\bigcap_{i=1}^{n}\left\{\omega: I_{i}(\omega)=a_{i}\right\}\right), \\
P(b) & =I_{\Omega}\left\{\omega: I_{1}(\omega)=b_{1}, \ldots, I_{n}(\omega)=b_{n}\right\} \\
& =P_{\Omega}\left(\bigcap_{i=1}^{n}\left\{\omega: l_{i}(\omega)=b_{i}\right\}\right) .
\end{aligned}
$$

Since $b \neq a, a$ and $b$ must differ in at least one coordinate $k$, and so $a_{k} \neq b_{k}$. Hence, $\xi_{\omega}: l_{k}(\omega)=a_{k} !$

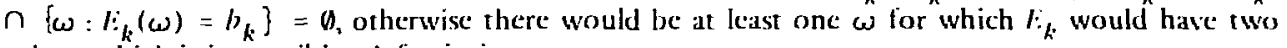
values, which is impossible. $A$ fortiori,

$$
\bigcap_{i=1}^{n}\left(\left\{\omega: l_{i}(\omega)=a_{i}\right\} \bigcap_{i=1}^{n}\left\{\omega: l_{i}(\omega)=b_{i}\right\}\right)=\emptyset .
$$

since subsets of disjoint sets are disjoint. Therefore,

$$
\begin{aligned}
\operatorname{Prob}(a \vee b) & =P_{\Omega}\left(\bigcap_{i=1}^{n}\left\{\omega: l_{i}(\omega)=a_{i}\right\} \bigcap_{i=1}^{n}\left\{\omega: l_{i}(\omega)=b_{i}\right\}\right) \\
& =P_{\Omega}\left(\bigcap_{i=1}^{n}\left\{\omega: E_{i}(\omega)=a_{i}\right\}\right)+P_{\Omega}\left(\bigcap_{i=1}^{n}\left\{\omega: E_{i}(\omega)=b_{i}\right\}\right) \\
& =P(a)+P(b) .
\end{aligned}
$$

In conclusion, whether we are discussing the four Div sets above, or the cvent sets in SVAM or SAA, the probability of any of these sets is simply the sum of the probabilities of their element (event) sets. For instance,

$$
P\left((\text { Div })_{d=0}\right)=\sum_{\left(u, \omega_{D}, \omega_{R}\right) \in(\text { Div })_{d=0}} P\left(u, \omega_{D}, \omega_{R}\right) .
$$

In addition, independence is simply not required in order to obtain a convenient and exact performance measure for a safeguard system, as we show in Ref. 7. In the present case, it suffices to add the probabilities of the appropriate sequences (or event sets). What may be a significant problem with the measures proposed in Ref. 10, however, is that the mutual exclusivity of the system state sequences precludes their independence, as we show in a related paper ${ }_{-}^{13}$ This result is not merely a matter of modeling, but is demanded by consistency requirements in the theory of quantitative probability. Hence, the form 


$$
P\left(\bigcup_{i=1}^{\infty} x_{i}\right)=1-\prod_{i=1}^{\infty}(1-P(1,))
$$

is inapplicable in any case.

Independence could play a more reasonable role in computing the probabilities of individual sequence triples $\left(u, \omega_{N}, \omega_{R}\right)$, ar "event sets," however. If the sequence members $\left.u, l\right)$-state $\omega_{J}$, and $k$-stare $\omega_{K}$ are mutually independent, then

$$
\left.P\left(u, \omega_{n}, . \omega_{k}\right)=P_{u}(u) P_{! !}^{\prime}\right)\left(\omega_{l}\right) P_{\Omega \Omega}\left(\omega_{R}\right)
$$

and we get

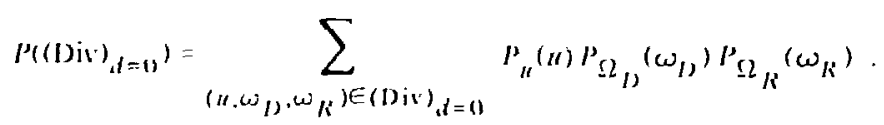

This could be the calse where the adversary is unaware ol the state $\left(\omega_{D}, \omega_{R}\right)$ of the system, and subsystems $D$ and $R$ fail independently.

A further simplification results if the elements of the vectors ("basic events") are themselves mutually independent, but this condition is much less likely to hold, even approximately. If the adversary is rational, and hecause of physical properties associated with the plant "real estate," any action ${ }_{k}$, in the scyuence " will strongly depend on the preceding action $u_{k-1}$ and on the succeeding $u_{k+1}$. The knowledge of some $u_{k}$ in a scquence $u$ may, in fact, determine the knowledge of all of $u$, on the lasis of dominance, for instance. Furthermore, any detection state component $\omega_{j}$ will depend on other components, particularly if common-mode conditions are considered. Nevertheless, if all these cumponent events were independent, we would obtain the form

$$
P\left((\text { Div })_{d=0}\right)=\sum_{\left(n, \omega_{D}, \omega_{R}\right) \in \mathrm{Div}} \prod_{d=0}^{n} P_{U_{i}}^{\left(u_{i}\right)} \prod_{j=1}^{m} P_{\Omega_{D}}\left(\omega_{D_{j}}\right) \prod_{k=1}^{p} P_{\Omega_{R_{k}}}\left(\omega_{R_{k}}\right) .
$$

In conclusion, if adversary and plant operating descriptions are complete, the measures $M_{i}$, $i=1,2,3,4$, proposed in Ref. 10 are inadequate* because, on the one hand, they poorly exploit the particular structure of the safeguard system and, on the other hand, they make unreasonable assumptions about it. Furthermore, in practical cases, the analyst cannot tell how well these measures approximate the true value of $P$ (Div), and this considerably weakens the defensibility of any probability analysis associated with these measures.

\subsection{PROBABILISTIC PERFORMANCE MEASURES ASSOCIATED WITH SAFEGUARD SYSTEMS}

If the diversion sequences do not include adversary acts of any kind, then the union of those sequences leading to diversion is a reasonable measure of system performance or, more accurately, system unreliability. If adversary acts are included, then other performance criteria must be contemplated, and these give rise to other measures. ${ }^{7}$

"The notation used in Ref. 10 is also wrong, since the statement " $K_{j} \in S_{i}$ " doesn't malic sense. The $K_{j}$ 's are probabilities and the $S_{i}$ 's are sets of events. 
Two probabilistic performance measures can be developed on the hasis of the deterministic outputs produced by SVAM. We assume that all diversion states are disjoint, but not necessarily independent, of course, since disjointness often implies dependence.

Because we do not include adversary acts, we take the two sets (Div) $d=0$ and (I) iv) $_{d=1}$ developed in Chapter 5 and nosdify them appropriately. We obtain three new sets

$$
\begin{aligned}
& \text { (1)iv) } \left.d=0, U=6=\left\{\left(\omega_{1}, \omega_{R}\right) \in \Omega_{y}, \times \Omega_{R}:(l)\left(\omega_{l}\right), \omega_{R}\right) \in\left(R^{-1}\left(w_{2}\right)\right\}_{d=0}\right\} \text {. } \\
& \text { (1)iv) })_{d=1, U=\emptyset}=\left\{\left(\omega_{l}, \omega_{K}\right) \in \Omega_{l} \times \Omega_{K}:\left\{()\left(\omega_{l}\right), \omega_{K}\right) \in\left(R^{1}\left(w_{2}\right)\right)_{d=1}\right\} \text {, }
\end{aligned}
$$

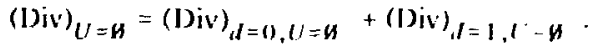

Using these sets, we obtain threc probabilistic performance measures $\left.\left.\left.\right|_{1} ^{\prime}: S \rightarrow \mid 0,1\right\} . \Psi_{2}^{\prime}: S \rightarrow \mid 0.1\right\}$. and $\Phi_{3}^{\prime}: S \rightarrow[0,1]$, defined for any safeguard system $S C ; \in S$ as follows:

$$
M_{1}^{\prime}: w_{3}^{\prime}(S C i)=P(\text { (I)iv) } d=0, U=w
$$

This is the unreliability measure for the detection subsystem $/$ ).

$$
M_{2}^{\prime}: \omega_{2}^{\prime}(\mathrm{SG})=P(\text { Div })_{d=1, U=u}
$$

This is an unreliability measure for the response subsystem $R$.

$$
M_{3}^{\prime}: \omega_{3}^{\prime}(\mathrm{SG})=P(\text { Div })_{U=0} \text {. }
$$

This is an unreliability measure for the system as a whole.

Because elements of (Div) $d=0, U=0$ and (Div) $d=1, U=0$ are mutually exclusice, the following representations are valid:

$$
\begin{aligned}
& \Phi_{1}^{\prime}(\mathrm{SG})=\sum_{\left(\omega_{D}, \omega_{R}\right) \in(\text { Div })_{d=0, U=n}} P\left(\omega_{D}, \omega_{R}\right), \\
& \Phi_{2}^{\prime}(\mathrm{SG})=\sum_{\left(\omega_{D}, \omega_{R}\right) \in(\text { Div })_{d=1, U=n}} P\left(\omega_{D}, \omega_{R}\right) .
\end{aligned}
$$

If states $\omega_{D}$ and $\omega_{R}$ and their components are statistically independent, they can be simplified further in the obvious manner.

The third measure $\Phi_{3}^{\prime}$, expressed as a structure function $\Phi_{3}^{\prime}\left(\Phi_{1}^{\prime}, \Phi_{2}^{\prime}\right)$ (sec Chapter 2) of the subsystem measures $\Phi_{1}^{\prime}$ and $\Phi_{2}^{\prime}$, assumes the very simple additive form

$$
\Phi_{3}^{\prime}(\mathrm{SG})=\Phi_{1}^{\prime}(\mathrm{SG})+\Phi_{2}^{\prime}(\mathrm{SG}) \text {, }
$$

the sum of the subsystem performance measures. Observe that this additive decomposition is valid whether complete system descriptions are used or "don't care" components are allowed. This is because the decomposition is based on the disjointness of the sets (Div) $d=0$ and (Div) $d=1$, and no other conditions are necessary.

As a concluding comment, it should be noted that because of the large number of "event sets" in the diversion sets $S_{1}, S_{2}, S_{3}, S_{4}$, SVAM does not, at this time, include a practical computer 
algurithm to produce correct reliability values or probabilities of successful diversion for these sets. During the research reported in this paper, this computational problem was analyzed, and we have produced an efficient technique. alled STOP (superfast technique for obtaining probabilities). This technique is reported in Ref. 7. 


\section{DETERMINISTIC COMPARISON OF SVAM AND SAA IN OUR FRAMEWORK}

In this chapter, we prove a short lemma which shows the equivalence between one of the basic outputs of SVAM, (Div) $l=0$, and the outputs of $S A A_{I}$. Then we further compare SVAM and $S A A_{I}$ in our franework for safeguard systens whose response subsystem $R$ works perfectly. This assumption is required because $\$ \wedge \Lambda_{1}$ deals with detection only, and not with response.

Our two-sulssystem model is repeated here for convenicnec as $1.7 g$. 8-1. Since tampering is not considered, the detection subsystem is $D: U \times \Omega_{l} \rightarrow V$, and the response subsystem is $R: V \times \Omega_{k} \rightarrow W$. To discuss $S A A_{1}$ in terms of our framework, recall our definition $S A \Lambda_{1}=$ L. $A, E, \Omega, M, M A P, T S, M O n, M O N\rangle$, where all the terms are as defined in Chapter 6 . In the $S \wedge \Lambda_{I}$ franework, two basic sequences describe the interaction of an adversary with a facility: the "aceessegress" sequence ("path") $u^{\prime}=\left(u_{1}^{\prime}, \ldots, u_{n}^{\prime}\right)$, and the material flow sequence $q=\left(q_{1}, q_{2}, \ldots, q_{u}\right)$. The target set selection function TS associates with each adversary $A$, material access point $m$, and plant state $\omega$ a pair of sequences of points in $I:,\left(u^{\prime}, q\right)=\operatorname{TS}(A, m, \omega)$. With each of these sequences is associated a possibly empty set of monitors $\left.\operatorname{MON}_{\omega}^{-1}\left(u^{*}, q\right\}\right) \subset$ Mon. For this analysis, there is no point in distinguishing between monitors associated with $q$-points $q_{j}$ or $u^{\prime}$-points $u_{i}^{\prime}$, and we can concatenate $u^{\prime}$ and $q$ into a single sequence $u=\left(u_{1}^{\prime}, \ldots, u_{n}^{\prime}, q_{1}, \ldots, q_{m}\right)$, which we relabel as $u=\left(u_{1}\right.$, $\ldots, u_{n}$ ). Tbese target sequences are tbe inputs to our system. The target sequence function TS may then be re-expressed as

$$
\text { IS : . } \$ 1 \times 1 \times \Omega \rightarrow l:
$$

and the input space is

$$
U=\{u=\operatorname{TS}(A, m, \omega):(A, m, \omega) \in . \mathscr{A} \times M \times \Omega\} .
$$

Next, $S \wedge \Lambda_{1}$ deals with binary detection only, not with response. As a detection subsystem, $S A A_{I}$ can thus be characterized by the function

$$
D_{1}: U_{1} \times \Omega \rightarrow V
$$

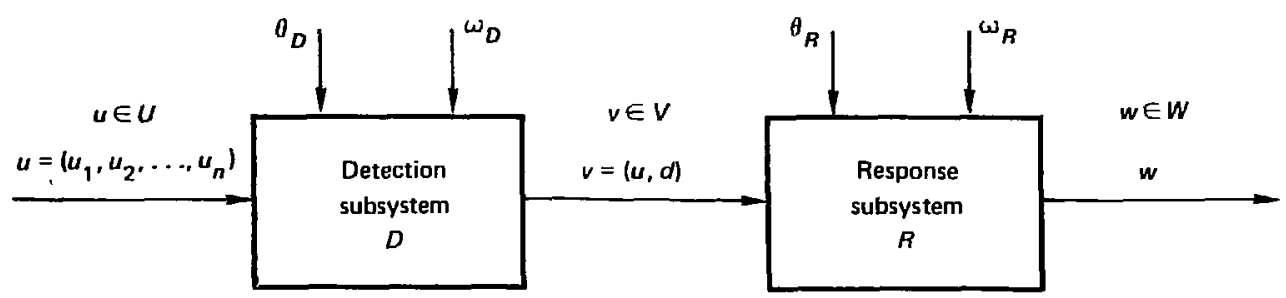

\section{Adversary Action Sequences $u$}

Action-Detection

Pairs $(u, d)$

Responses

Fig. 8-1. An aggregated model of a safeguard system. Inputs are adversary action $u$, tampering acts $\left(\theta_{D}, \theta_{R}\right)$, and plant state $\left(\omega_{D}, \omega_{R}\right)$. Outputs arc detection outputs $v$ and diversion outcomes $w$. (Repeat of Fig. 3-3.) 
defined by

$$
b_{l}(u, \omega)=(u, d)_{\omega}=\left(\left(u_{1}, u_{2}, \ldots, u_{n}\right),\left(d_{1}, \ldots, d_{n}\right)\right)_{\omega} .
$$

where

$$
d_{i}=1 \text { iff } \mathrm{MON}_{\omega}^{-1}\left(\left\{u_{i}\right\}\right) \neq: \emptyset, i=1,2, \ldots, n .
$$

In words, for all target sequences $u$ and states $\omega$, the detection subsystem assigns $d_{i}=I$ to those elements ${ }_{i}$ of $"$ which are in the ficld of view of at least one monitor. Thus any sequence $a$ is detected ift at least one of its elements is within reach of at least one monitor.

Now recall that the principal output of $S \wedge \Lambda_{I}$ is the monitor null set of a safeguard system (for a given set of states $\omega \in \Omega$ ). This is

$$
\text { Null }=\bigcup_{\omega \in \Omega} \operatorname{Null}(\omega)
$$

where

$$
\operatorname{Nulk}(\omega)=\bigcup_{A \in \mathscr{A}}\left[t=\operatorname{TS}(A, m, \omega): \operatorname{MON}_{\omega}^{-1}([t])=\emptyset, m \in \operatorname{MAP}(A, \omega)\right]
$$

Because we want to compare $S A A_{1}$ and SVAM in our framework, and SAA, only deals with detection, we consider only those safeguard systems whose response subsystems are known to work perfectly. For such systenss (no tampering), the response function $R: V \times \Omega_{R} \rightarrow W^{\prime}$ is defined by $R\left(v, \omega_{R}\right)=w_{2}$ (diversion) iff there docs not cxist a $d_{i}$ in $v=\left(\left(v_{1}, v_{2}, \ldots, v_{n}\right),\left(d_{1}, \ldots, d_{n}\right)\right)$ such that $d_{i}>0$. We call such an $R$ a perfect response subsystem.* For such a system, only undetected sequences can lead to diversion. (Recall that we do not consider false alarms in this report.) The perfection of $\mathrm{R}$ obviously depends on the definit.on of its input and output spaces $V$ and $W$. For more complicated spaces, $W$-spaces with many $w_{2}$-values and $V$-spaces with false alarms, for instance, criteria for perfection would also be more complex.

In Chapter 5, we defined a set

$$
\text { (Div) }{ }_{d=0}=\left[\left(u, \omega_{D}, \omega_{R}\right):\left(D\left(u, \omega_{D}\right), \omega_{R}\right) \in\left(R^{-i}\left(w_{2}\right)\right)_{d=0}\right\} \text {. }
$$

These were the undetected sequences of acts $u$ and states $\left(\omega_{D}, \omega_{R}\right)$ which led to diversion. Now we show that, when the response subsystem $R$ is known to be perfect, (Div) $d=0$ and Null are exactly the same. ${ }^{t}$

Lemma. For a sa: iguard system with perfect response subsystem $R$, the sets $\left(\mathrm{Div}_{d=0}\right.$ and ivill are the same.

Proof. To show that (Div) ${ }_{d=0}=$ Null, it suffices to show that each is contained in the other, or that every element of one is an element of the other.

Fix some $\omega \in \Omega$. Take any $t \in \operatorname{Null}(\omega)$. Then

$$
\bigcup_{c \in\{t\}} \operatorname{MON}_{\omega}^{-1}(\{e\})=\varnothing
$$

* Recall that we are dealing with diversion acts only, not legitimate acts. Hence, there are no false alarms.

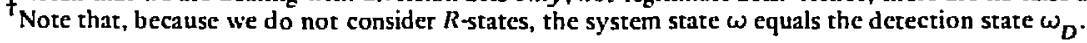


But this implies that

$$
D(t, \omega)=\left(\left(t_{1}, \ldots, t_{n}\right),\left(d_{1}=0, d_{2}=0 \ldots, d_{n}=0\right)\right) .
$$

Hence,

$$
l(t, \omega) \in\left(K^{1}\left(w_{2}\right)\right)_{d=0} \text { (recall that } \Omega_{k} \text { is not applicable) }
$$

Hence,

$$
\left.(t, \omega) \in(I)^{\prime}\right)_{d} d=0
$$

To prove the converse, take any $(u, \omega) \in\left(\right.$ Div) $d=0$. Then $l(u, \omega) \in\left(R^{-1}\left(w_{2}\right)\right)_{d=0}$ (no $\left.\Omega_{k}\right)$. But then $l(u, \omega)=\left(\left(u_{1}, \ldots, u_{n}\right),(0,0, \ldots, 0)\right)$, and $\bigcup_{r \in\{j} \operatorname{MON}_{\omega}^{-1}(\{e\})=0$. Hence $u \in$ Null $(\omega)$, and thus, since $\omega \in \Omega, u \in$ Null. Therefore Null $=($ (l)iv) $d=0$. Q.E.D.

Since the set (biv) $d_{l=0}$ is the same as $S_{1}$ of $S V \Lambda M$, if we assume a perfect response subsystem, the set $N u$ ull produced by $S \wedge \Lambda_{J}$ is the same as $S_{f}$ as well. Outputs of $S V A M$ and $S A \Lambda_{I}$ are thus basically the sance if $R$ is perfect. If not, the various sets $S_{1}, S_{2}, S_{3}, S_{4}$ of SVAM produce* additional input-output information about $R$.

We have confined our comparison to the deterministic aspects of both methods. While $S A A$ produces probabilistic computations at Level II and at higher levels, SVAM is not designed to produce such outputs at this timc, and no comparison was made on statistical grounds.

- Assuming our interpretation of those secs. 


\section{CONCLUSIONS}

The nuclear safeguard problem addressed at Lawrence Livermorc Laboratory is a complex large-scale systems problem involving many uncertainties. If this problem is addressed as a systems problem, it is important to have a clear understanding of what a safeguard system is. We have contributed towards such an understanding by developing the formal notion of a safeguard system as a parametrized input-output concept whose inputs are scenarios contemplated or attempted by any of a class of admissible adversaries, and whose outputs are possible consequences or outcomes of diversion attempts. The parameters may be viewed as design factors and states of nature (environmental or failure conditions, operating modes, ...) which influence the internal structure of the system. In this report, we have developed a simple but formal vicw of a safeguard system as consisting of two basic subsystems: the detection subsystem $(D)$, and the response subsystem $(R)$, and we have precisely formulated a host of iniportant safeguard questions based on this decomposition. Because some of these questions are probabilistic, and the language and usage of probability theory are not universal in the enginecring business, we have carefully introduced the important concepts of probability theory to clarify our approach.

Much safeguards work has been accomplished at Lawrence Livermore Laboratory, and two methodologies have emerged in the past few years: the structured assessment approach (SAA), and the systems vulnerability assessment metiod (SVAM). Both these methods have been formally described in our framework, and compared, and some previously developed system performance measures were structured in accordance with our two-subsystem decomposition. One probabilistic measure acquired a particularly simple structure. The probability of successful diversion was shown to decompose into the sum of two measures associated with subsystems $D$ and $R$, respectively.

\section{ACKNOWLEDGMENTS}

It would have been impossiblc to write this report without interactions with several members of the safeguards program. Such interactions were essential in testing the results reported here, and in surrounding these results with appropriate examples.

I am most indebted to Alfred Parziale who, as always, was paticnt enough to listen to my rantings and, because of his familiarity with the overall safeguards program, helped me stay on a practical and applied course. My thanks go also to Richard Schechter, who played a similar role and came up with clever counterexamples which I was too optimistic to consider.

Sincere thanks are owed to R. Al-Ayat and W. F. Rousseau for reviewing this report prior to publication, and for providing helpful comments.

Finally, we must acknowledge the efforts of Cathy Tyree, Bernice Farmer, and Louise Harlan, who patiently deciphered our handwriting and typed the whole thing in record time. 


\section{REFERENCES}

1. G. C. Corynen, A lioundation for Structuring the Safeguards System, witb Specific l:mphasis on tbe Notion of a Structural livent, Lawrence Livermore Laboratory, Livermore, Calif., Nuclear Safeguards Program Report MC-79-195 (June 1979).

2. Iः. L. Crane, W. D. Altman, W. B. Brown, R. J. Dube, J. W. Hockert, D. S. Thurman, L. I'. Wirfs, and D. B. Matthews, Report of tbe Material Control and Material Acconnting Task Force, U. S. Nuckar Regulatory Commission, Washington, D. C., NUREC-0450 ( $\wedge$ pril 1978), Vols. I-V.

3. G. C. Corynen, A Matbematical Tbeory of Modeling, Lawrence Livermore Laboratory, Livermore, Calif., UCIRL-51726 (December 1974).

4. R. E. Barlow and F. Proschan, Statistical Theory of Reliability and Life Testing (I.lolt, Rinchart and Winston, New York, 1975).

5. L. Brciman, Prohability (Addison-Wesley, Reading, Mass., 1968).

6. K. L. Chung, A Course in Probahility Tbcory (Academic Press, New York, 1974).

7. (i. C. Corynen, A New Tecbnique for Computing tbe Probability of Complex livents, Lawrence Livermore Laboratory, Livermore, Calif., Nuclear Waste Management Report WM-79-672 (November 1979).

8. T. R. Rice and S, L. Derby, Overview of tbe Structured Assessment Approacb and Documentation of Algoritbms to Compute tbe Probability of Adversary Detection. Applied Decision Analysis, Inc., Menlo Park, Calif., for Lawrence Livermore Laboratory, UCRL-13937 (December 1978).

9. A. A. Parzialc, I. J. Sacks, T. R. Rice, and S. L. Derby, Tbe Structured Assessment Aulysis of Facility $X$, Lawrence Livermore Laboratory, Livermore, Calif., Nuclear Safeguards Program Report MC-79-12 (January 1979), Vol. I, Laecutive Summary.

10. J. J. Lim, Safeguards System Lffectiveness Measures to Date, Lawrence Livermore Laboratory. Livermore, Calif., Nuclear Salfeguards Program Memorandum MC-79-74 (1979).

11. J. J. Lim, Lawrence Livermore Laboratory, Livermore, Calif., private communication (lebruary 1979 ).

12. S. Rai, "An Efficient Method for Reliability Evaluation of a General Network," IEl:F: Trans. Reliability R-27, 206-211 (1978).

13. G. C. Corynen, On the Assessment of the Probability of a Diversion Attempt, Lawrence Livermorc Laboratory, Livermore, Calif., Nuclear Safeguards Program Memorandum MC-79-124 (1979). 


\section{APPENDIX. THE SAFEGUARD SYSTEM AS A STRUCTURED OBJECT}

In this appendix, we show how our model of the safeguard system (Fig. 3-3. Chapter 3) fits into the general framework developed in Ref. 1. The basic concept in that framework is the notion of a structured system, a triple $S=\langle$ PIS, PIOS, $\Theta\rangle$, where

$$
\operatorname{PIS}=\left\{\mathbb{P}_{t}, \boldsymbol{P}_{0}, \boldsymbol{P}_{\mathrm{S}}, \mathrm{PIM}\right\}
$$

the parametrized intercomection structure of $S$, and

$$
\begin{aligned}
& \Phi_{1}: \Theta \rightarrow . \mathbb{K}_{1} \text { is the frammetrized imput intercemmection structure, } \\
& P_{O}: \Theta \rightarrow \mathscr{H}_{O} \text { is the parametrized ontput intercomnection structure. } \\
& \mathscr{P}_{S}: \Theta \rightarrow \cdot \mathscr{H}_{\mathrm{S}} \text { is the parametrized subsystem interconmection stracture, } \\
& \mathrm{PIM}=\left\{\Theta_{,} \Theta_{l}, E_{\mathrm{Pl}} . E_{\mathrm{Pl}}\right\} \text {, the parameter interconnection matixi. }
\end{aligned}
$$

Furthermore,

$$
\begin{aligned}
& \text { PIOS }=\left[\mathrm{PIOS}_{i}: i \in I\right\} \cup\left[\mathrm{PIOS}_{S}\right] \text { is the purametrized input-output (I/O) structure of } S \text {, } \\
& \operatorname{PIOS}_{i}: \Theta_{i} \rightarrow 2^{U}{ }^{X Y_{i}}{ }_{i} \text { is the } \Theta_{i} \text {-parametrised input-output cbaracteristic }{ }^{*} \text { of subsystem } i \in I \text {, } \\
& \operatorname{PIOS}_{S}: \Theta \rightarrow 2^{U \times Y} \text { is the } \Theta \text {-parametrized sy'stem input-output cbaracteristic of } S \text {. }
\end{aligned}
$$

Our safeguard model (Fig. $A-1$ ) is a simple example of such a system. For this model, the input, output, and subsystem interconnection structures are simply the matrices

$$
\begin{gathered}
!_{1}, \\
\text { IIM }=U\left[\begin{array}{l}
1 \\
W
\end{array},\right.
\end{gathered}
$$

and

$$
\operatorname{SIM}=w_{1}\left[\begin{array}{cccc}
U_{1} & V & \Theta_{D} \times \Omega_{D} & \Theta_{R} \times \Omega_{R} \\
0 & 1 & 0 & 0 \\
0 & 0 & 0 & 0
\end{array}\right]
$$

respectively.

\footnotetext{
*Note the distinction between claracteristic and structure.
} 


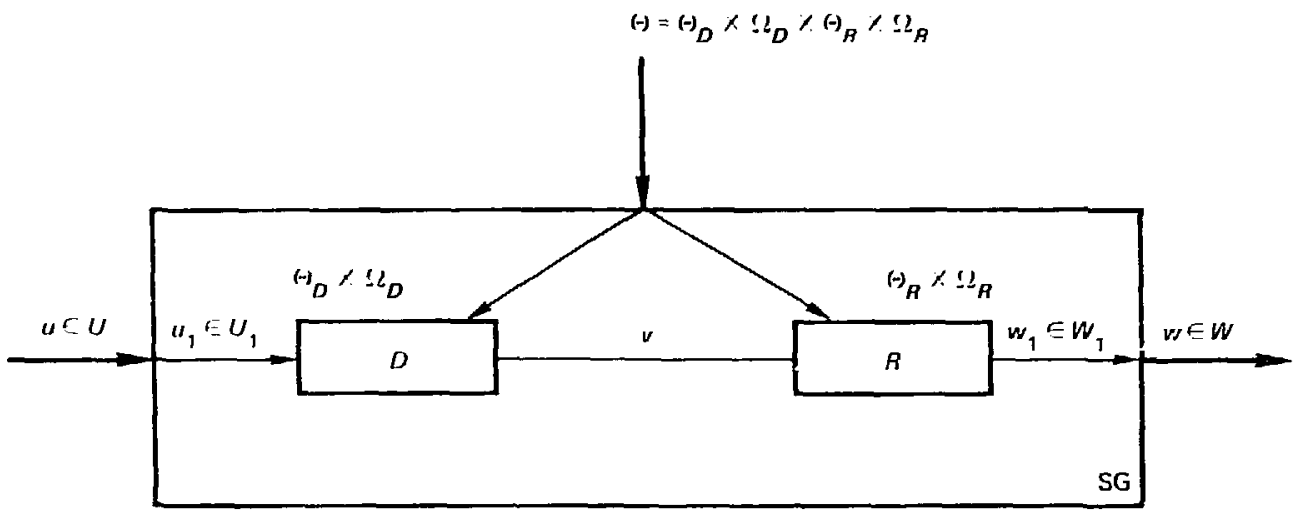

Fig. A-1. An aggregated safeguard system as a structured parametrized system.

The parameter interconnection matrix ${ }^{1}$ is

$$
\left.\left.\operatorname{PIM}=\left\langle(-)_{,(-)}, \times \Omega_{l,},()_{k} \times \Omega_{k}\right), \mathrm{PRO}\right), \&_{\mathrm{PROJ}}\right\rangle .
$$

where PROJ consists of two projection maps*

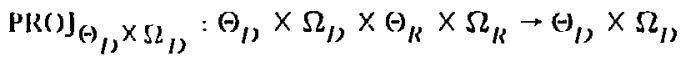

and

$$
\operatorname{PRO} \mathrm{J}_{(-)} \times \Omega_{R}:()_{1}, \times \Omega_{l} \times()_{R} \times \Omega_{R} \rightarrow \Theta_{R} \times \Omega_{R} .
$$

Next,

$$
\mathscr{E}_{\text {PROJ }}:\{\Theta\} \times\left\{\Theta_{D} \times \Omega_{I}, \Theta_{R} \times \Omega_{R}\right\} \rightarrow \text { PROJ }
$$

associates

$$
\operatorname{PROJ}_{\Theta_{D}} \times \Omega_{D} \text { with }\left(\Theta, \Theta_{D} \times \Omega_{D}\right) \text {, }
$$

and

$$
\mathrm{PROJ}_{\Theta_{R}} \times \Omega_{R} \text { with }\left(\Theta, \Theta_{R} \times \Omega_{R}\right) \text {. }
$$

\footnotetext{
*A two-dimensional projection map is defined as follows (similarly for higher dimensions: PROJ $_{X}: \mathrm{X} \times \mathrm{V} \rightarrow \mathrm{X}$

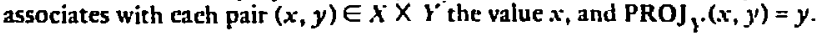


The parametrized input-output structure is

$$
\text { PIOS }=\left\{\operatorname{PIOS}_{D}, \operatorname{PIOS}_{R}, \operatorname{PIOS}_{\mathrm{SG}}\right\} \text {. }
$$

where

$$
\operatorname{PIOS}_{D}: \Theta_{D} \times \Omega_{D} \rightarrow L^{\prime} \times V^{\prime}
$$

the purametrized input-output detection function, associates with each $\left(\theta_{D}+\omega_{D}\right) \in \Theta_{D} \times \Omega_{i}$, the input-output detection function

$$
D\left(\cdot, \theta_{n}, \omega_{l}\right): U \rightarrow V
$$

obtained from

$$
D: U \times \Theta_{I} \times \Omega \rightarrow V
$$

by fixing $\theta_{D}$ and $\omega_{D}$, and

$$
\operatorname{PIOS}_{R}: \Theta_{R} \times \Omega_{R} \rightarrow V \times W^{\prime}
$$

the parametrized input-output response function, associates with each $\left(\theta_{R}, \omega_{R}\right) \in \Theta_{R} \times \Omega_{R}$ the $1 / 0$ response functionn

$$
R\left(\bullet, \theta_{R}, \omega_{R}\right): V \rightarrow H
$$

and

$$
\operatorname{PIOS}_{S G}: \Theta \rightarrow W^{\prime}
$$

the parametriacd imput-output safeguard function, associates with each $0 \in \Theta$ the safcguard inputoutput function

$$
\mathrm{SG}(\bullet, 0): U \rightarrow W
$$

